\title{
A New Polarized Neutron Interferometry Facility at the NCNR
}

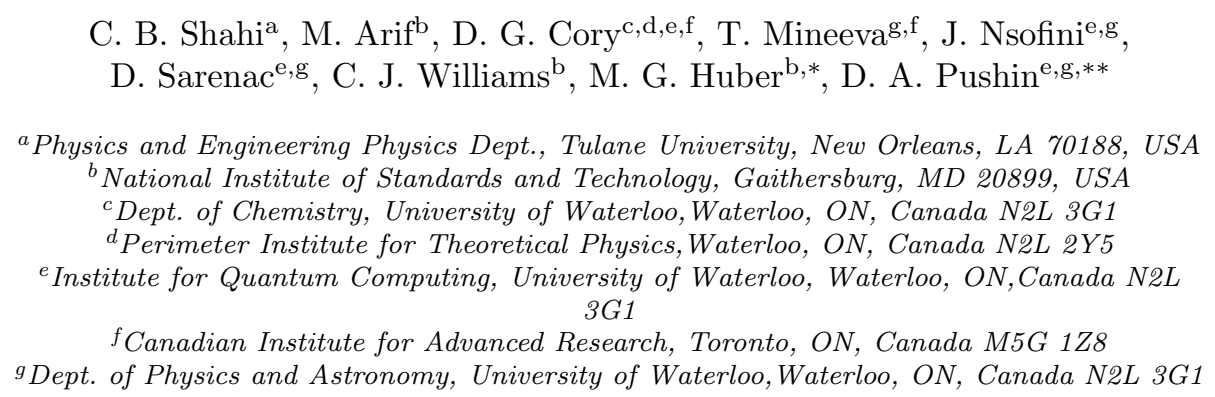

\section{Abstract}

A new monochromatic beamline and facility has been installed at the National Institute of Standards and Technology (NIST) Center for Neutron Research (NCNR) devoted to neutron interferometry in the research areas of spin control, spin manipulation, quantum mechanics, quantum information science, spintronics, and material science. This facility is possible in-part because of advances in decoherence free subspace interferometer designs that have demonstrated consistent contrast in the presence of vibrational noise; a major environmental constraint that has prevented neutron interferometry from being applied at other neutron facilities. Neutron interferometry measures the phase difference between a neutron wave function propagating along two spatially separated paths. It is a practical example of self interference and due to its modest path separation of a few centimeters allows the insertion of samples and macroscopic neutron spin rotators. Phase shifts can be caused by gravitational, magnetic and nuclear interactions as well as purely quantum mechanical effects making interferometer a robust tool in neutron research. This new facility is located in the guide hall of the NCNR upstream of the existing Neutron Interferometer and Optics Facility (NIOF) and has several advantages over the NIOF including higher incident flux, better neutron polarization, and increased accessibility.

\footnotetext{
${ }^{*}$ Corresponding author

${ }^{* *}$ Corresponding author

Email addresses: michael.huber@nist.gov (M. G. Huber), dmitry.pushin@uwaterloo.ca (D. A. Pushin)
} 
The long term goal for the new facility is to be a user supported beamline and make neutron interferometer more generally available to the scientific community. This paper addresses both the capabilities and characteristics of the new beamline.

Keywords: Neutron interferometry; Polarized neutrons; Quantum information science

\section{Introduction}

The National Institute of Standards and Technology (NIST) Center for Neutron Research (NCNR) located in the Gaithersburg, MD is a user-focused facility [1] that provides thermal and cold neutrons beams for a wide variety of applications. These include materials science, the study of polymers, biological science, neutron standards and dosimetry studies, and fundamental physics research. Neutrons are used in research applications because they interact differently than light/electron beam techniques, can deeply penetrate materials, have wavelengths that coincide with material lattice spacing, exhibit isotope-dependent scattering that is especially strong in the case of hydrogen, and through their magnetic dipole moment interact with magnetic materials

For many of these research areas cold neutrons which have an energy below $25 \mathrm{meV}$ (corresponding to wavelengths $\lambda$ longer than $0.18 \mathrm{~nm}$ ) are preferable. To maximize cold neutron flux, thermal neutrons with $\lambda<0.18 \mathrm{~nm}$ produced by the reactor are cooled by a liquid hydrogen (LH) cold source [2]. A large number of the instruments at the NCNR are located in the guide hall where the cold neutrons leaving the LH source have been transported tens of meters using evacuated neutron guides (NG). With the addition of a new interferometry beamline, the Neutron Interferometry and Optics Facilities (NIOFs) are now the second and third instruments located downstream of the cold source on NG-7. The location of both interferometer facilities are highlighted in Fig. 1 that shows the layout of the NCNR. The neutron interferometry (NI) program at the NCNR has a history of successful measurements of nuclear constants and 


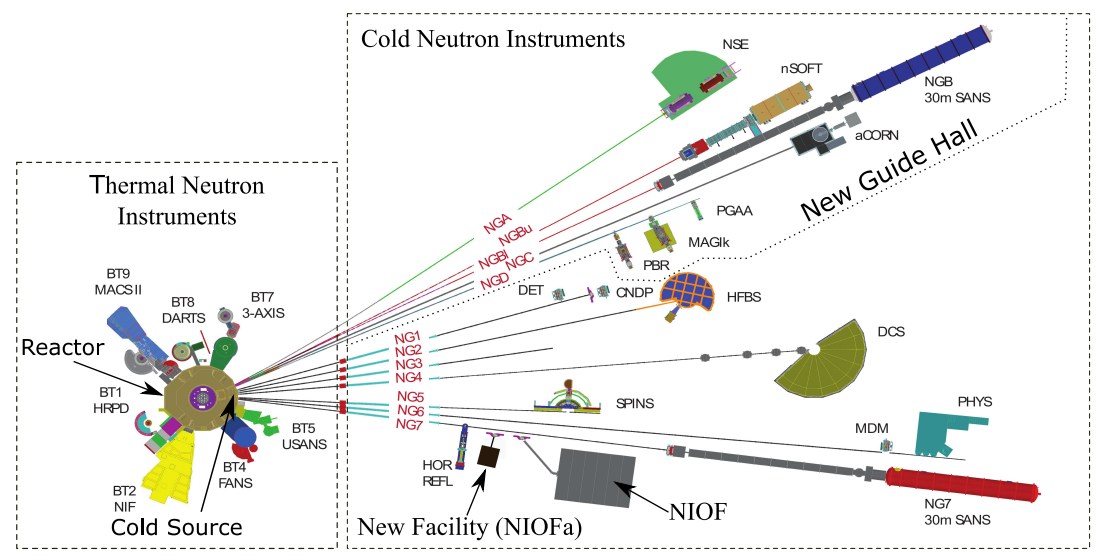

Figure 1: Layout of the NCNR. The reactor core (purple) is surrounded by several instruments (labeled as BT\#) requiring higher energy neutrons. The cold source (hidden) is located at the intersection of the NG guides. The NIOFa beamline resides on NG7 and is upstream of existing beamline NIOF.

39 neutron phase effects (see Table 1). fisherThese include demonstration of the $404 \pi$ periodicity of neutron wave function [3], precision coherent and incoherent 41 scattering length measurements [4-7], measuring vertical coherence [8], demon42 strating contrast in the presence of zero entanglement but non-zero Quantum

${ }_{43}$ Discord [9], and more applied experiments including phase contrast imaging ${ }_{44}[10,11]$ and measuring the density of thin films [12]. More recently, proposed 45 experiments have needed both high neutron polarization and precise spin ma46 nipulation. Although polarized neutrons have been used at the NIOF it is in 47 some ways not ideally suited for these types of experiments. One obstacle in 48 neutron interferometry is its sensitivity to the environment. The path length in49 side a neutron interferometer is on the order of $10 \mathrm{~cm}$ long and it takes a neutron 50 approximately $50 \mu$ s to travel that distance over which it undergoes greater than ${ }_{51} 10^{8}$ oscillations. This relatively long (compare to light that in the same time can travel $15 \mathrm{~km}$ ) flight time is the reason why neutron interferometers are extremely sensitive to external disturbances such as thermal instabilities, thermal gradients, vibrational noise, and acoustical noise. To preserve long-term phase stability at the older NIOF, the neutron interferometer is housed in a massive, 
thermal, acoustical, and vibration isolated hutch (see Fig. 2) that has its own foundation separate from the rest of the building [21]. The hutch constrains the neutron interferometer $9 \mathrm{~m}$ away from the neutron guide and this contributes to the low neutron rates in the detector on the order of $10 \mathrm{~s}^{-1}$. When using neutron polarizers that have a maximum transmission of $40 \%$ this count rate

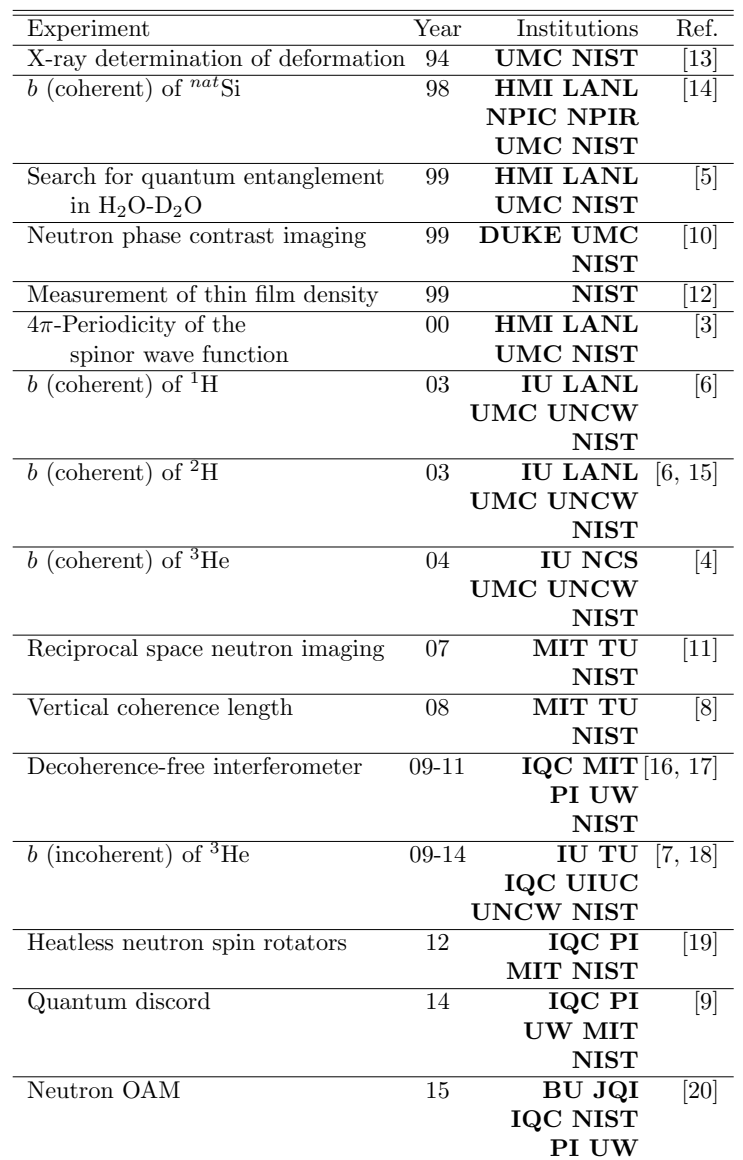

Table 1: List of NI experiments done at or with the collaboration of the National Institute of Standards and Technology (NIST). Institutions are Boston University (BU), Duke University (DUKE), Indiana University (IU), Institute for Quantum Computing (IQC), Joint Quantum Institute (JQI), Los Alamos National Laboratory (LANL), Massachusetts Institute of Technology (MIT), University Missouri-Columbia (UMC), Nuclear Physics Institute Czech (NPIC), Nuclear Physics Institute Russia (NPIR), Perimeter Institute for Theoretical Physics (PI), University North Carolina-Wilmington (UNCW), University of Waterloo (UW), University of Illinois at Urbana-Champaign (UIUC), University of Maryland (UMD), and Tulane University (TU). 


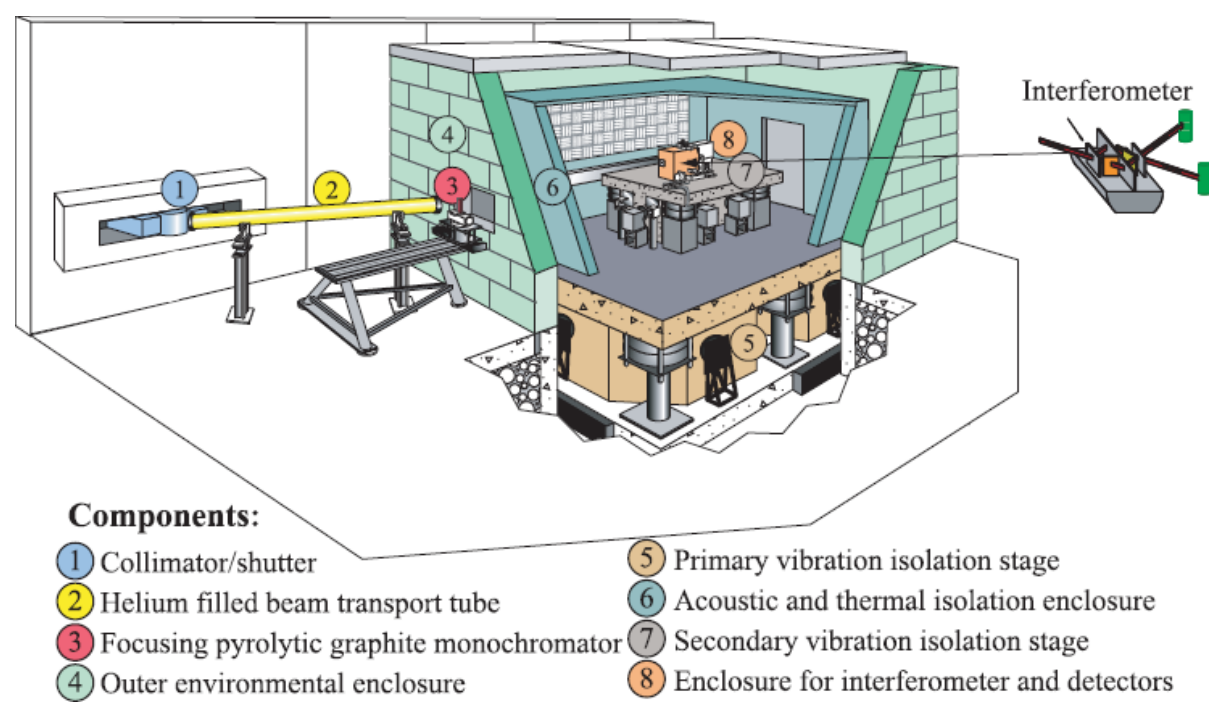

Figure 2: An artist's depiction of the hutch at the NIOF showing the nested-layers and vibration isolation system.

drops even further. To compensate for poor initial statistics many NI experiments need to be run for many months and insuring experimental (especially environmental) conditions over that long span of time can be challenging.

In 2010 the NCNR underwent an expansion project adding an additional guide hall with four new state-of-the-art neutron guides (see Fig. 1). During the yearlong reactor shutdown required for this expansion, a new beamline just upstream of the NIOF was installed. This new facility, designated as NIOFa, benefits from higher flux at the expense of some long-term phase stability. Construction of this facility is in-part due to the recent advances in neutron interferometer design. A decoherence free subspace (DFS) neutron interferometer has shown to be insensitive to vibrational frequencies up to $22 \mathrm{~Hz}$ [16]. The new facility has improved neutron polarimetry capabilities, increased ease-of-access, a designated cryostat, and environmental temperature control. Further it will expand the capabilities, some of which are not feasible in the NIOF beamline, of the neutron interferometry program at NIST. The new facility will focus on: spin-based neutron interferometry, testing of quantum mechanics, spintronics, neutron spin manipulation, material research and quantum information science. 
${ }_{78}$ This paper covers aspects and characteristics of the new NIOFa facility. For improved understanding of NI applications this includes a general treatment of spin-based neutron interferometry. Detailing the changes to the older beamline, see the companion paper to this one [22].

\section{Neutron Interferometry}

\subsection{Neutron Optics}

Neutron optics exploits the wave-like properties of neutrons and describes the neutrons interaction with materials. In general it is analogous to optics describing light propagation and shares many of the same concepts like diffraction, interference, coherence, and refraction (see Table 2). When a neutron with initial momentum $k_{0}=\sqrt{2 m_{n} E_{0}} / \hbar$ passes through a medium (as depicted in Fig. 3) its momentum changes to $K=n k_{0}$. Here $E_{0}$ is initial energy, $m_{n}$ the mass of the neutron and $n$ is the index of refraction which one can write as

$$
n=\frac{K}{k_{0}}=\sqrt{\frac{2 m_{n}\left(E_{0}-V\right)}{2 m_{n} E_{0}}} \approx 1-\frac{V}{2 E_{0}} .
$$

91 The potential $V$ can represent any neutron interaction as the neutron couples to the strong, weak, electromagnetic and gravitational forces. The approximation in Eqn. (1) is valid because the effective potential for neutrons is $\approx 10^{-6} E_{0}$.

\begin{tabular}{lcr} 
Property & Photon & Neutron \\
\hline \hline Mass & 0 & $m_{n}=1.674928(1) \times 10^{-27} \mathrm{~kg}$ \\
Spin & $\hbar$ & $\frac{1}{2} \hbar$ \\
Magnetic Dipole Moment & 0 & $\mu_{n}=-9.66237055(71) \times 10^{-27} \mathrm{~J} / \mathrm{T}$ \\
Equation & $\nabla^{2} \psi(\boldsymbol{r}, t)=\frac{1}{c} \frac{\delta^{2} \psi(\boldsymbol{r}, t)}{\delta^{2} t}$ & $H \psi(\boldsymbol{r}, t)=i \hbar \frac{\delta \psi(\boldsymbol{r}, t)}{\delta t}$ \\
Energy & $E=\hbar k c$ & $E=\frac{\hbar^{2} k^{2}}{2 m_{n}}$ \\
$n\left(\mathrm{H}_{2} \mathrm{O}\right)-1$ & 0.33 & $\mathrm{v}=\frac{3956}{\lambda[m]} \times 10^{-10} \mathrm{~m} / \mathrm{s}$ \\
Velocity & $c$ & $E=m_{n} c^{2}=939.6 \mathrm{MeV}$
\end{tabular}

Table 2: Comparison between light and neutron properties. A similar table can be found in [23]. Values are taken from the latest PDG summaries [24]. $n\left(\mathrm{H}_{2} \mathrm{O}\right)$ is the index of refraction for water. Calculated values for the neutron are expressed for the thermal neutron wavelength of $0.18 \mathrm{~nm}$. 


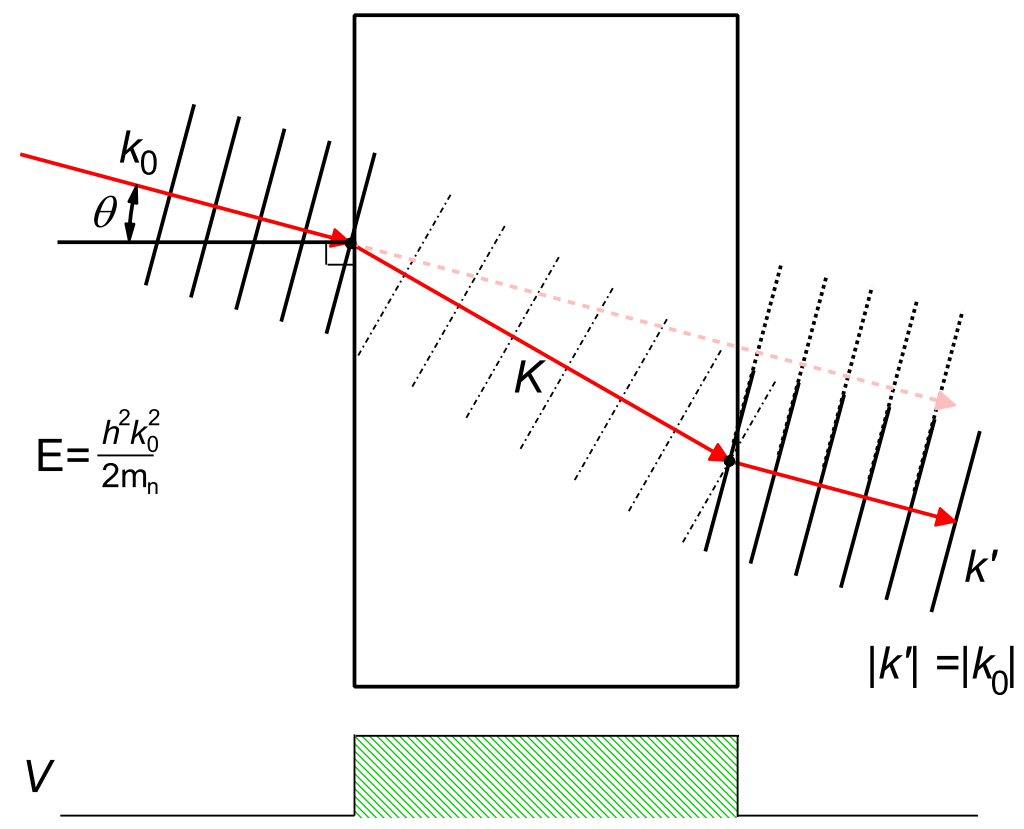

Figure 3: A neutron passing through a medium can be described by an index of refraction $n$ and potential $V$ changing the incident wavevector $k_{0}$ to $K$. The angle of refraction is exaggerated as $n \approx 1$.

94 Note that one of the other major differences between light and neutron optics,

95 other than the magnitude of $n$, is that

$$
n=\frac{K}{k_{0}} \propto \frac{1}{v_{\text {med }}} \quad \text { (photon) }
$$

96 and

$$
n=\frac{K}{k_{0}} \propto v_{\text {med }} \quad \text { (neutron) }
$$

${ }_{97}$ where $v_{\text {med }}$ is the velocity inside the medium. The relevant potential for a 98 neutron interaction with a sample material is called the Fermi pseudopotential 
and is given by

$$
V_{\text {nuc }}=\frac{2 \pi \hbar^{2}}{m_{n}} N b
$$

where $N$ is the atomic density and $b$ is the scattering length. The scattering length is a property of the interaction, varies per isotope, and is typically on the order of a few femtometers. Further, $b$ is a complex number where $b=b^{\prime}+i b^{\prime \prime}$. The scattering cross section $\sigma_{s}$ is given simply by $\sigma_{s}=4 \pi|b|^{2}$. The imaginary part $b^{\prime \prime}$ is related to the neutron absorption cross section

$$
\sigma_{a}=\frac{4 \pi}{k_{0}} b^{\prime \prime} .
$$

Neutron absorption in a material can be described by the transmission of a neutron beam with an incident intensity $I_{0}[25]$ by

$$
I=I_{0} e^{-\sigma_{a} N D}
$$

The real part of neutron scattering lengths are overwhelmingly positive with some notable exceptions like ${ }^{1} \mathrm{H},{ }^{48} \mathrm{Ti}$, and ${ }^{62} \mathrm{Ni}$. One of the primary means of measuring $b$ has been NI which has measured over fifty isotopes (many to high precision).

\subsection{Interferometry}

A single crystal neutron interferometer consists of a silicon crystal that has been machined to produce several parallel blades on a common base. This ensures arcsecond alignment of lattice planes of each blade relative to each other; a stringent requirement of Bragg diffraction. Shown in Fig. 4 are the interferometers available at NIST. Properties for these interferometers are listed in Table 3. A single crystal neutron interferometer is conceptually analogous to an optical Mach-Zehnder interferometer. However, instead of mirrors a neutron 
interferometer uses beam splitters and Bragg diffraction defined by

$$
\mathrm{n} \lambda=2 d \sin \theta_{B}
$$

to separate the neutron into 2 paths. It is important to note that this separation happens for each individual neutron's wavefunction (counts rates are low enough to guarantee that here is only one neutron at a time inside the interferometer).

In Eqn. $7 \mathrm{n}$ is the order of reflection, $d$ is the lattice spacing of the crystal and $\theta_{B}$ is the Bragg angle. For silicon $d=(0.543 \mathrm{~nm}) / \sqrt{h^{2}+l^{2}+k^{2}}[26]$ where $h, k, l$ are the Miller indices for a particular lattice. Silicon has a diamond-structure lattice and many reflections are forbidden. Valid reflections are

$$
h, k, l=\left\{\begin{array}{l}
\text { all odd } \\
\text { all even with } h+k+l=4 \mathrm{~m}
\end{array}\right.
$$

where $\mathrm{m}$ is an integer. The most widely used lattice planes are (220) and (111). Wavelengths used for neutron interferometry experiments at NIST fall between $0.2 \mathrm{~nm}$ and $0.5 \mathrm{~nm}$. The most commonly used are $0.271 \mathrm{~nm}$ and $0.235 \mathrm{~nm}$.

Figure 6 shows a schematic of a neutron interferometer. A neutron entering the first blade (splitter) of the interferometer is coherently split into two spatially separated paths labeled as path I and path II. Neutrons along path I and path II are again Bragg diffracted by the middle blade(s) (mirror) and interfere

\begin{tabular}{rccccccr}
\hline \hline & $\begin{array}{c}\text { Lattice } \\
\text { Type }\end{array}$ & $\begin{array}{c}\lambda \text { Range } \\
(\mathrm{nm})\end{array}$ & $\begin{array}{c}\text { Max. Contrast } \\
(\%)\end{array}$ & $\begin{array}{c}B_{w} \\
(\mathrm{~mm})\end{array}$ & $\begin{array}{c}B_{h} \\
(\mathrm{~mm})\end{array}$ & $\begin{array}{c}B_{s 1} \\
(\mathrm{~mm})\end{array}$ & $\begin{array}{r}B_{s 2} \\
(\mathrm{~mm})\end{array}$ \\
\hline 1 Skew & $(111)$ & $0.33-0.49$ & - & 2.5 & 30.0 & 20.0 & 36.6 \\
2 Skew & $(111)$ & $0.22-0.30$ & 34 & 1.5 & 40.0 & 14.1 & 82.0 \\
3 Skew & $(220)$ & $1.00-0.30$ & - & 2.0 & 30.0 & 32.0 & 25.4 \\
4 LLL & $(111)$ & $0.00-0.50$ & - & 2.0 & 25.0 & 41.5 & - \\
5 LLL & $(111)$ & $0.00-0.50$ & 90 & 2.5 & 30.4 & 39.6 & - \\
6 LLL & $(220)$ & $0.00-0.20$ & - & 2.5 & 15.5 & 34.5 & - \\
7 Skew & $(220)$ & $0.22-0.25$ & 85 & 2.8 & 25.4 & 16.0 & 30.5 \\
8 DFS & $(220)$ & $0.00-0.33$ & 25 & 1.0 & 16.0 & 10.0 & 20.0 \\
9 LLL & $(111)$ & $0.00-0.54$ & 30 & 1.0 & 15.0 & 10.0 & -
\end{tabular}

Table 3: Properties of the interferometers located at NIST shown in Fig. 4. Geometry blade width, height and separation are defined in Fig. 5. The maximum contrast is the largest value that has been observed for any wavelength. Interferometers with contrasts unspecified have either not been used in the last 15 years at NIST and/or are believed to be $<10 \%$. 


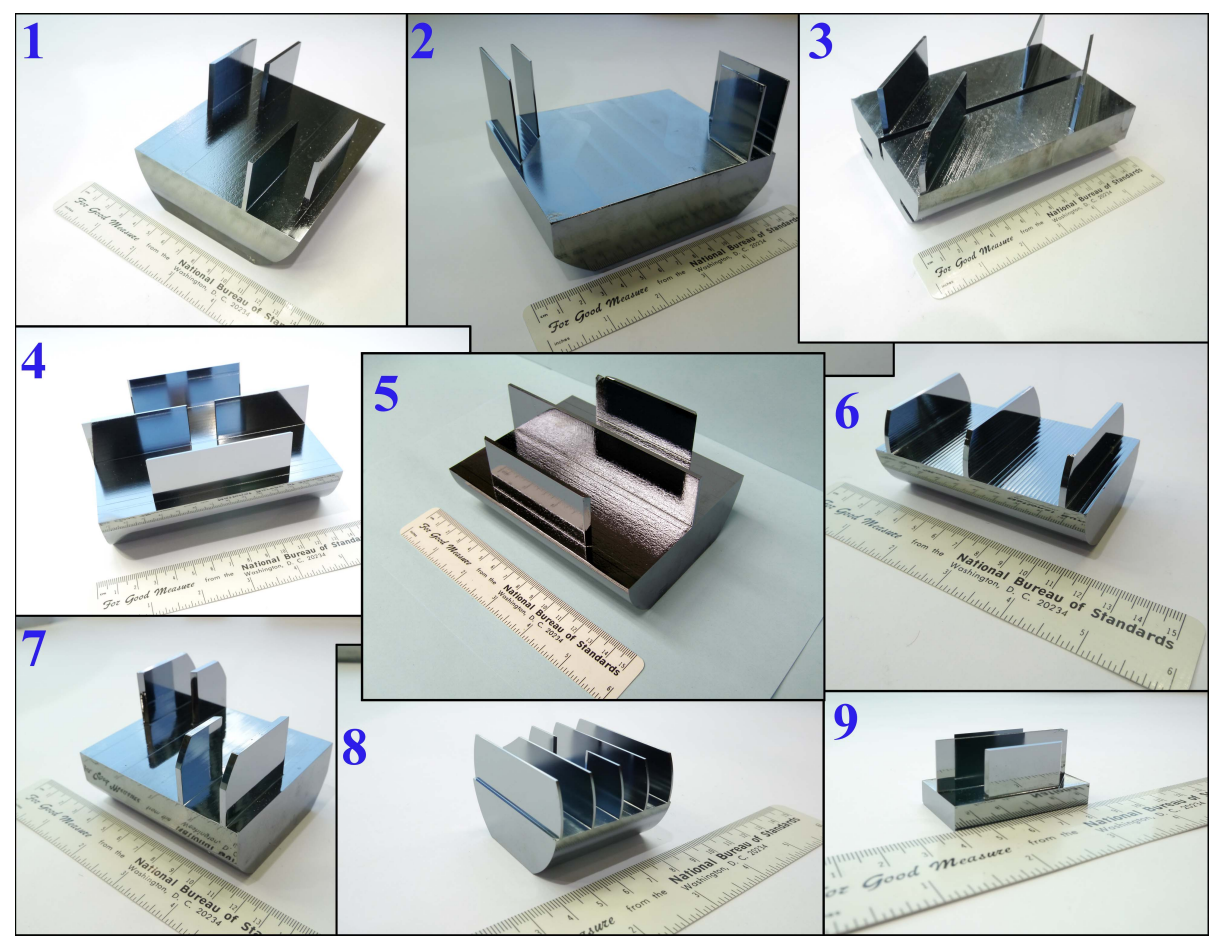

Figure 4: The single crystal interferometers at NIST (formerly the National Bureau of Standards). Their properties are given in Table 3. Many of the interferometers shown here were fabricated by C. Holmes and S. Potts at the U. of Missouri-Columbia machine shop under the supervision of S. A. Werner.

in the final blade (analyzer) of the interferometer. The two beams exiting the interferometer are historically labeled the O- and H-beams. Neutrons are detected with $100 \%$ efficiency by cylindrical ${ }^{3} \mathrm{He}$ filled proportional counters. The intensities measured by the detectors can be written as

$$
\begin{aligned}
I_{O} & =A[1+C \cos (\Delta \phi)] \\
I_{H} & =A[B-C \cos (\Delta \phi)] .
\end{aligned}
$$

In principle, the values of the parameters $A, B$, and $C$ can be determined by calculation of the reflection and transmission coefficients along the 2 paths as the neutron Bragg diffracts in the silicon crystal. In practice, however, these parameters are extracted from fits to the data. Notice that in the interferometer 


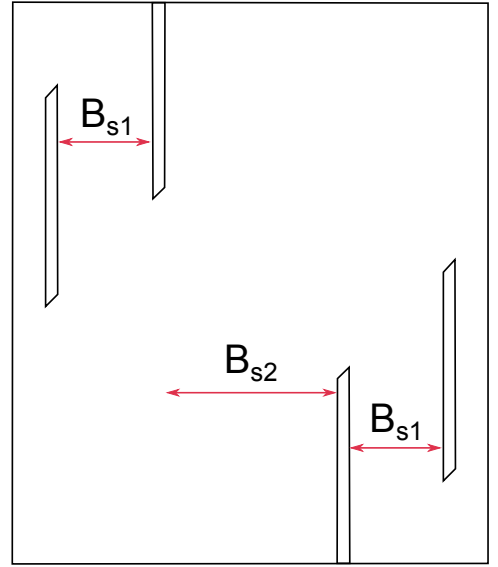

(a)

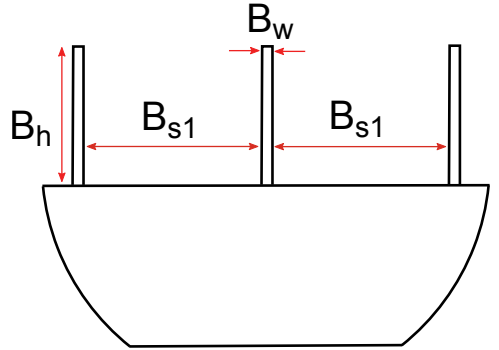

(b)

Figure 5: Geometries of a skew and LLL neutron interferometer. (a) Top view of skew symmetric neutron interferometer, where $B_{s 1}$ and $B_{s 2}$ (for the case of Skew and DFS geometries) are the blade separations. (b) Side view of a LLL interferometer showing blade height $B_{h}$, width $B_{w}$, and separation $B_{s 1}$.

$I_{O}+I_{H}=$ constant as neutrons are not absorbed in the silicon crystal. The phase $\Delta \phi$ is path-integral phase difference between the two paths. In practice $\Delta \phi=\Delta \phi_{\mathrm{V}}+\Delta \phi_{0}$ where $\Delta \phi_{\mathrm{V}}$ is the phase shift caused by a sample (or other interaction). For instance, when the phase shift $\Delta \phi_{\mathrm{V}}$ is caused by a sample located in path 2 one may write

$$
\begin{aligned}
\Delta \phi_{\text {sample }} & =\int_{\mathrm{I}} k_{0} d s-\int_{\mathrm{II}} n k_{0} d s \\
& \approx \int_{\mathrm{II}} \frac{V}{2 E_{0}} d s=\int_{\mathrm{II}} N b \lambda d s=-N b \lambda D
\end{aligned}
$$

where $N, b$, and $D$ are number density, scattering length and the effective thickness of the sample. The phase $\Delta \phi_{0}$ is the an initial phase difference between the paths that can vary depending on experimental conditions. If the sample absorbs neutrons then Eq. 8 is modified using Eqns. 6 as

$$
I_{O}=\frac{A}{2}\left[\left(1+e^{-\sigma_{a} N D}\right)+2 e^{-\sigma_{a} N D / 2} C \cos (\Delta \phi)\right]
$$

151 Although the amplitude of $I_{H}$ transforms in the same way as in Eq. 11, to 


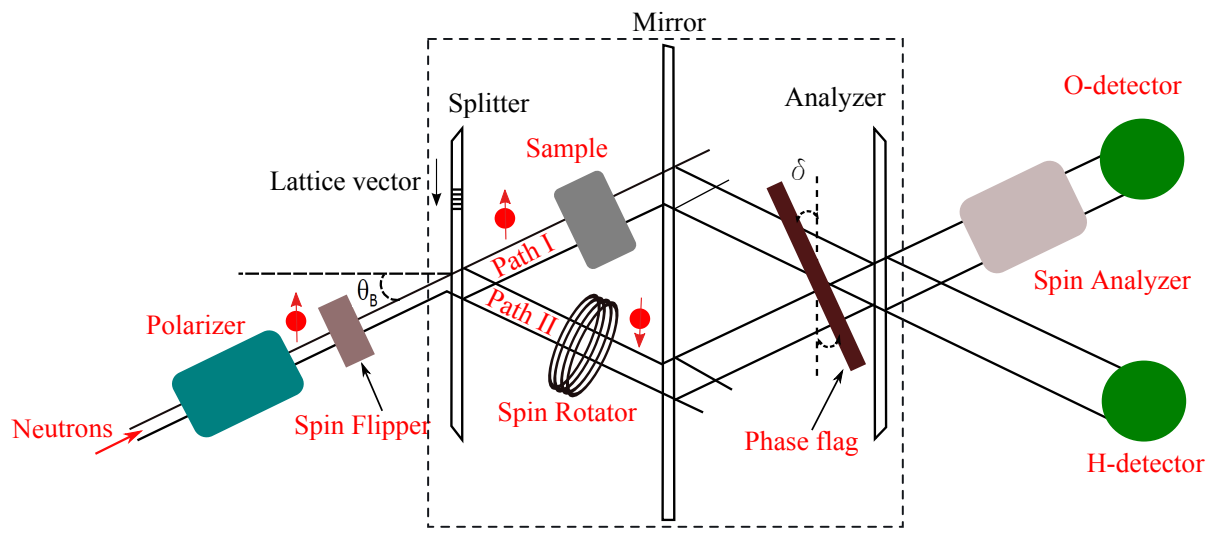

Figure 6: A diagram of a three blade interferometer. The incident neutron beam, which can be polarized in either spin state using a supermirror and precession coil spin flipper, enters the interferometer from the left. The 'splitter' blade of the crystal separates the neutron into 2 separate paths which are reflected using a 'mirror' blade toward the 'analyzer' blade where they interfere with each other. The neutron intensity is measured via proportional ${ }^{3} \mathrm{He}$ detectors.

transform the $B$ term in Eq. 9 it would become necessary to express it as individual reflection and transmission coefficients; a complication that is omitted here.

In addition to the interaction to be probed $\left(\Delta \phi_{\mathrm{V}}\right)$ and the intrinsic phase of the interferometer $\left(\Delta \phi_{0}\right)$ one often adds a variable 'control' sample called a phase flag. At the Neutron Interferometer and Optics Facilities (NIOFs) the phase flag is a $50 \mathrm{~mm} \times 50 \mathrm{~mm} \times 1.5 \mathrm{~mm}$ optically flat piece of fused quartz. By rotating the phase flag, one creates a phase shift due to the geometrical difference in optical path lengths between the paths of

$$
\begin{aligned}
\Delta \phi_{\text {p.f. }}(\delta) & =-N_{\text {p.f. }} b_{\text {p.f. }} \lambda D_{\text {p.f. }}\left[\frac{1}{\cos \left(\theta_{B}+\delta\right)}-\frac{1}{\cos \left(\theta_{B}-\delta\right)}\right] \\
& =-N_{\text {p.f. }} b_{\text {p.f. }} \lambda D_{\text {p.f. }}\left[\frac{2 \sin (\delta) \sin \left(\theta_{B}\right)}{\cos ^{2}\left(\theta_{B}\right)-\sin ^{2}(\delta)}\right]
\end{aligned}
$$

where $N_{\text {p.f. }}, D_{\text {p.f. }}$, and $b_{\text {p.f. }}$ are the number density, thickness, and average scattering length of the phase flag, respectively. The angle $\delta$ is the angle of phase flag from its initial position parallel to the blades (see Fig. 6). Rotating a phase flag a few degrees produces interferograms like the ones seen in Fig. 7 
and allows a fitted determination of each of the coefficients given in Eqns. 8 and 9.

The neutron is spin $1 / 2$ particle with a magnetic dipole moment (MDM) of $\mu_{n}=-9.66 \times 10^{-27} \mathrm{~J} / \mathrm{T}$ (the minus sign indicates that the neutron's spin and MDM are anti-aligned). Thus the neutron can be polarized and analyzed using a variety of methods such as Stern-Gerlach magnets, supermirrors, polarized ${ }^{3}$ He spin filters, and Huelser crystals. Typically these devices reach neutron polarizations $P_{n}$ better than $90 \%$ for monochromatic neutron beams. Once polarized, the neutron's polarization can be manipulated with nearly $100 \%$ efficiency using standard neutron flippers of which there are many designs: RF coils [27, 28], DC driven precession spin flippers [29, 30], and thin films [19] to name a few. The phase shift caused by magnetic fields can be quite complicated as the overall phase shift is both dependent on the type (static vs time varying) and magnitude of the magnetic field as well as the specific spacial evolution of the neutron's spin around a spin sphere [30]. In its simplest form the magnetic potential

$$
V_{\mathrm{mag}}=-\mu_{n} \boldsymbol{\sigma}_{\boldsymbol{i}} \cdot \boldsymbol{B}
$$

is a function of Pauli matrices $\boldsymbol{\sigma}_{\boldsymbol{i}}$ and the magnetic field $\boldsymbol{B}$. When the neutron spin is aligned parallel or anti-parallel to the magnetic field, this potential produces a phase shift of

$$
\Delta \phi_{\operatorname{mag}}= \pm \frac{1}{\hbar} \int_{I I} \frac{\mu_{n} B}{2 E} d s= \pm \frac{2 \pi \mu_{n} \lambda m_{n} B D}{h^{2}}
$$

Besides magnetic and nuclear interactions, there are numerous other potentials, that are listed in Table 4, including gravity, Sagnac (Coriolis), and AharonovCasher. 


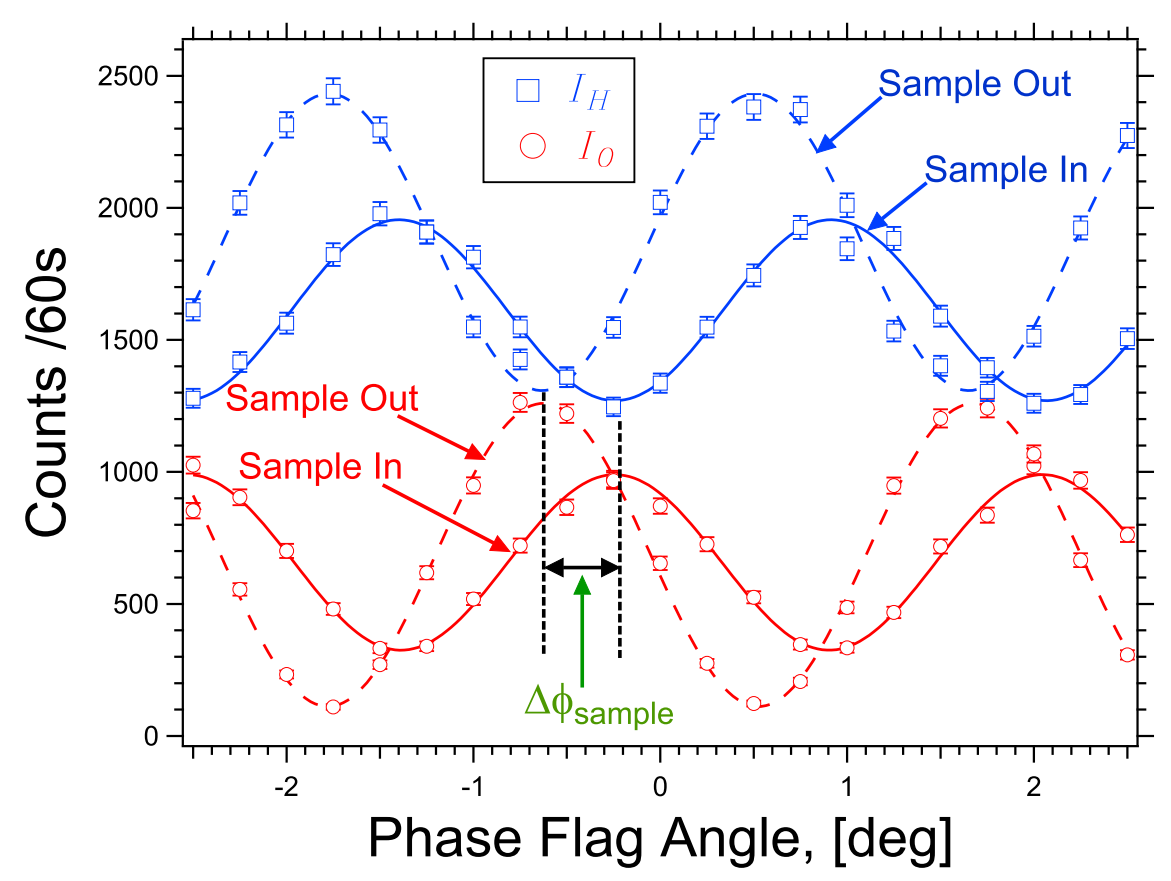

Figure 7: An interferogram showing $I_{O}$ (red circles) and $I_{H}$ (blue squares) where the lines are the fits using Eqs. $8 \& 9$. The phase shift $\Delta \phi_{\text {sample }}$ is measured comparing the sample in (solid) and out (dotted) positions for a sample with appreciable scattering losses. The uncertainties are purely statistical.

\section{NIOFa Facility Design}

\subsection{Guide modifications}

At the NCNR neutrons are produced in the reactor core by fission of ${ }^{235} \mathrm{U}$ and moderated to thermal energies using heavy water. Typically the reactor operates at full power for 38-day periods followed by 10 day maintenance breaks. For neutron instruments like the Neutron Interferometer and Optics Facility

\begin{tabular}{lccr} 
Interaction & Potential & Phase shift $[\phi]$ & Reference \\
\hline \hline Nuclear & $2 \pi \hbar^{2} b \delta(\boldsymbol{r}) / m_{\mathrm{n}}$ & $-N D \lambda b$ & {$[31]$} \\
Magnetic & $-\boldsymbol{\mu}_{\mathbf{n}} \cdot \boldsymbol{B}(\boldsymbol{r})$ & $\pm \mu_{\mathrm{n}} B m_{\mathrm{n}} \lambda D /\left(2 \pi \hbar^{2}\right)$ & {$[31]$} \\
Gravitational & $m_{\mathrm{n}} \boldsymbol{g} \cdot \boldsymbol{r}$ & $m_{\mathrm{n}}^{2} g \lambda A \sin (\alpha) /\left(2 \pi \hbar^{2}\right)$ & {$[32]$} \\
Coriolis & $-\hbar \boldsymbol{\omega} \cdot(\boldsymbol{r} \times \boldsymbol{k})$ & $\left(2 m_{\mathrm{n}} / \hbar\right) \boldsymbol{\omega} \cdot \boldsymbol{A}$ & {$[33]$} \\
Aharonov-Casher & $-\boldsymbol{\mu}_{\mathbf{n}} \cdot(\boldsymbol{v} \times \boldsymbol{E}) / c$ & $\pm\left\{2 \mu_{\mathrm{n}} /(\hbar c)\right\} \boldsymbol{E} \cdot \boldsymbol{D}$ & {$[34]$} \\
Scalar Aharonov-Bohm & $-\boldsymbol{\mu}_{\mathbf{n}} \cdot \boldsymbol{B}(t)$ & $\pm \mu_{\mathrm{n}} B t / \hbar$ & {$[35]$} \\
Berry & & $\Omega / 2$ & {$[36]$}
\end{tabular}

Table 4: The phase shift caused for many interactions. The definitions of the undefined symbols can be found in the references. (Table reproduced from [23]) 
(NIOF) located in the Guide Hall, these thermal neutrons pass through a liquid hydrogen (LH) cold source. The LH source decreases the peak of the MaxwellBoltzmann energy distribution to a temperature of $40 \mathrm{~K}$; thus becoming what are called cold neutrons ${ }^{1}$. Cold neutrons are then transported to the various instruments by rectangular neutron guides. The older guides labeled NG1 through NG7 are made of glass. The inside of the glass guides are coated with ${ }^{58} \mathrm{Ni}$. This allows for increased neutron transmission as ${ }^{58} \mathrm{Ni}$ has a relatively large critical angle of $\theta_{c}^{2}=0.34 \mathrm{mrad}$ per $\mathrm{meV}$ and thus minimizes neutron transport losses compare to naked glass. The NG7 guide, which houses both the NIOF and the new NIOFa, is internally $15 \mathrm{~cm}$ tall and $6 \mathrm{~cm}$ wide at the location of beam exit.

Before the upgrade, a single pyrolytic graphite $\mathrm{PG}(002)$ monochromator that was internal to the guide was used to provide neutrons to the NIOF hutch. A PG crystal, which has a hexagonal lattice structure, Bragg diffracts neutrons of the desired energy. The pyrolytic graphite crystals used at the NIOFa has an angular variation of $0.5^{\circ}$ in lattice vector direction; a feature that is otherwise known as a mosaic $\eta$. A mosaic crystal allows a greater intensity to be reflected relative to a perfect crystal where $\eta=0$.

For the new facility, a $150 \mathrm{~mm}$ portion of NG7 guide was removed and two pyrolytic graphite $\mathrm{PG}(002)$ monochromators were installed in the lower half of the NG7 guide (see Fig. 8). The upper half of the guide is reserved for a small angle neutron scattering (SANS) instrument located at the end position of NG7. The guide windows are magnesium foils $900 \mu \mathrm{m}$ thick. Both PG crystals are $50 \mathrm{~mm}$ tall by $40 \mathrm{~mm}$ wide. Since these monochromators along with their stages and supports are located outside of the guide, they can be more conveniently maintained. The two reflected beams from the monochromators cross as the upstream monochromator provides neutrons for the NIOF and downstream monochromator for the NIOFa. At the NIOFa beamline the PG monochromator provides a fixed $0.44 \mathrm{~nm}$ neutron beam $\left(\theta_{B}=41^{\circ}\right)$. This wavelength was

\footnotetext{
${ }^{1}$ This is higher than the LH temperature of $20 \mathrm{~K}$ since the neutrons are not in equilibrium with the cold source.
} 
chosen to maximize the neutron intensity as it is near the peak of the MaxwellBoltzmann distribution provided by the LH cold source.

The NIOFa was installed in an area upstream of the hutch; in a space that was reserved for the NIOF since its installation in 1994. This area was planned into the original NIOF design to allow for a large range of neutron wavelengths to be accessible. The NIOF uses a double monochromator system to reflect neutrons parallel to the neutron guide and into the hutch. Although the first monochromator is a fixed position relative to the guide; the second verticallyfocusing monochromator is on a $1.8 \mathrm{~m}$ travel translation stage. This translation stage can be kinematically placed along several positions on the floor allowing wavelengths from $0.2 \mathrm{~nm}$ with $\theta_{B}=17.3^{\circ}$ to $0.47 \mathrm{~nm}$ with $\theta_{B}=45.0^{\circ}$ (the relative angle of the reflected beam to the guide is $\left.2 \theta_{B}\right)$. However, with the addition of the new facility, the NIOF facility can only access neutrons from $0.2 \mathrm{~nm}$ to $0.31 \mathrm{~nm}$ without impacting the NIOFa beamline. The NIOF retains the capability of using $0.32 \mathrm{~nm}$ to $0.47 \mathrm{~nm}$ neutrons but in that case both facilities can not run simultaneously.

\subsection{Description of new beamline}

A schematic of the new beamline can be seen in Fig. 9. Cold $0.44 \mathrm{~nm}$ neutrons coming from reactor are reflected out of the NG7 guide by the downstream monochromator PG(002) labeled as Mon2. Both Mon1 and Mon2 are directly coupled to a nema true [37] planetary gearhead with 100:1 ratio and stepper motor $^{2}$. The output shafts of the motors are coupled to an incremental encoder $^{3}$ with 8192 counts per revolution. Tilt of the monochromators is achieved through motorized ${ }^{4}$ goniometers $^{5}$. In order to minimize activation of the crystal mounting, the PG crystals are mounted using a thin, slotted aluminum pieces, the top of which sit $2 \mathrm{~mm}$ below the guide.

\footnotetext{
2 model \# NT17-100-H-34-615-557-D769

3 model \# 15T-02SF-8192N5RHV-F00

${ }^{4}$ Faulhaber model \# AM1524-A-0.25-15.5-6 15/8 900:1

${ }^{5}$ model \# NT66-525 (Mon2) and model \# NT66-531 (Mon1)
} 


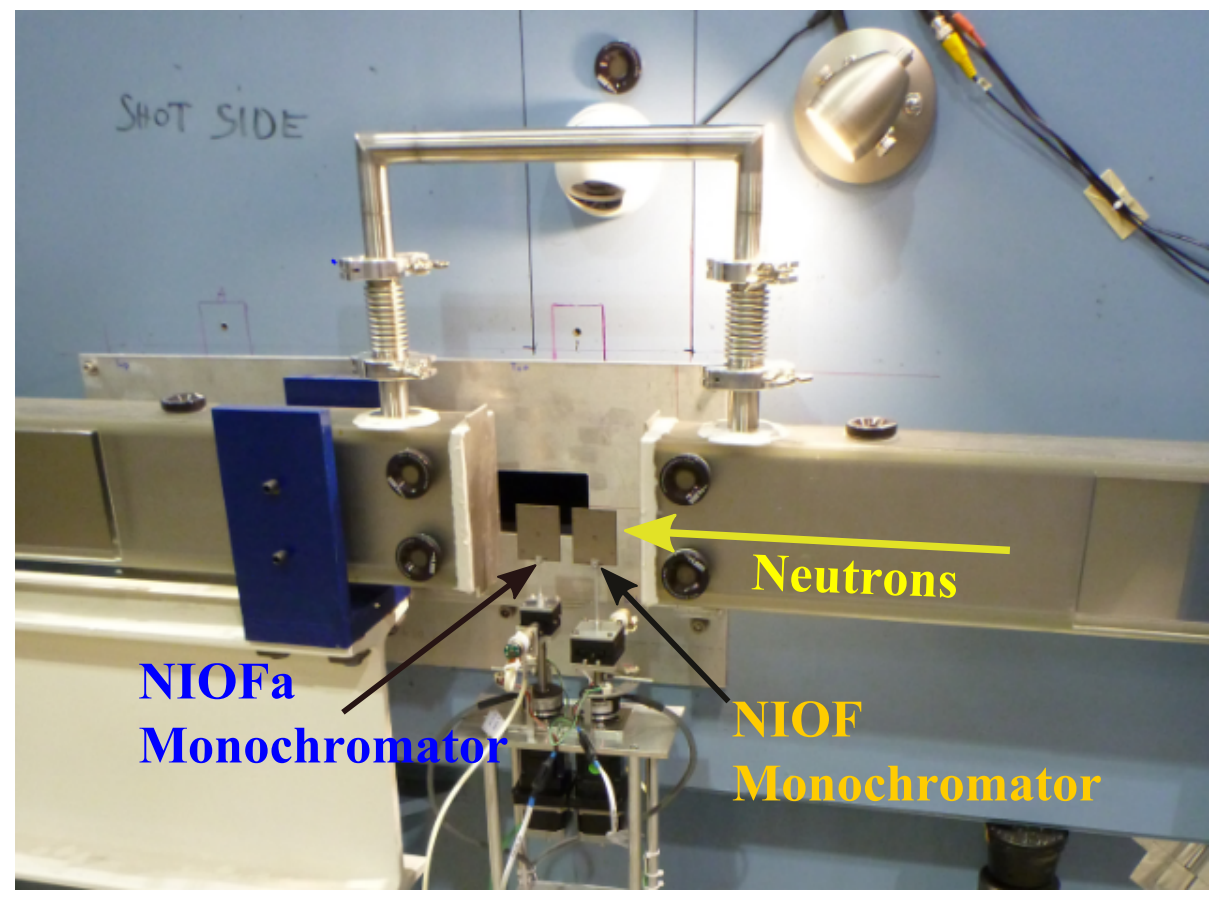

Figure 8: The current setup with a guide cut of $150 \mathrm{~mm}$ where there are two identical PG(002) monochromator crystals.

Paraffin wax and steel shot-filled-steel walls (the teal shielding in Fig. 9) surround the neutron guides for radiation safety and to minimize backgrounds. This shielding extends $1.98 \mathrm{~m}$ high and is approximately $318 \mathrm{~mm}$ thick in total. At the NIOF there is a $177 \mathrm{~mm}$ high by $571 \mathrm{~mm}$ long rectangular hole in the back (guide side) of the shielding centered on the beamline. The beamline itself is $120 \mathrm{~cm}$ from the floor. To reduce backgrounds, a $6.35 \mathrm{~mm}$-thick, neutronabsorbing boral plate was installed between the guide and the shielding. This boral plate has a $100 \mathrm{~mm}$ long by $62 \mathrm{~mm}$ high rectangular hole that allows neutrons from Mon1 and Mon2 to pass. Between the boral and the shielding wall is $50.8 \mathrm{~mm}$ of lead that has a slightly larger rectangular hole than the boral plate allowing neutrons to pass through unobstructed.

A $412.8 \mathrm{~mm}$ gap exists between the two shielding walls at the interferometer facility. This gap contains a rotatable collimator which is called the "Drum". The Drum was originally built with the NIOF and can be translated so that 
any wavelength between $0.2 \mathrm{~nm}$ to $0.47 \mathrm{~nm}$ could be selected for the hutch. To fill in the gap, two paraffin wax and steel-shot-filled steel "wings" were attached to sides of the drum and that translated with the drum as it moved for various wavelengths. With the addition of the NIOFa beamline the upstream most wing was removed.

Due to the large angles the exit hole on the downstream shield is $279 \mathrm{~mm}$ high by $1334 \mathrm{~mm}$ wide. A paraffin and steel insert with a $63.5 \mathrm{~mm}$ vertical gap was installed inside the $279 \mathrm{~mm}$ exit hole to reduce backgrounds at the facility. Since a large epithermal neutron background has been measured, a high-density polyethylene (HDPE) block was installed in the gap to downscatter neutrons so that they could be more efficiently captured on neutron shielding. A combination of $6.35 \mathrm{~mm}$ thick boron nitride $(\mathrm{BN})$ and $76.2 \mathrm{~mm}$ thick lead sits beyond the downstream shield wall. To limit horizontal beam divergence a $0.2^{\circ}$ Söller collimator [38] with neutron absorbing gadolinium-coated blades is inside the shielding upstream of the boron nitride and lead shielding.

To open and close the beam at the facility, a shutter is used containing $12.7 \mathrm{~mm}$ thick boron-nitride, $1.0 \mathrm{~mm}$ thick cadmium, and $101.6 \mathrm{~mm}$ of lead. The shutter is translated vertically in and out of the beam using air pistons ${ }^{6}$. A fission chamber ${ }^{7}$ with a detection efficiency of $10^{-5}$ is located after the shutter to monitor reactor fluctuations. Following the fission chamber there is an adjustable slit assembly labeled as Slit1 which is made of two orthogonal pairs of $3.2 \mathrm{~mm}$-thick boron-nitride-layered cadmium mounted on translational stages. Because there is a substantial component of higher energy neutrons in the NIOFa beamline (see Sec. 3.3.2) a $177.8 \mathrm{~mm}$ diameter, $234.95 \mathrm{~mm}$ long cylindrical dewar with a beryllium filter inside was installed after Silt1. The beryllium filter scatters neutrons with $\lambda<0.40 \mathrm{~nm}$ out of the direct beam where they are absorbed in the surrounding boron shielding. To minimize the transmission loss of the desired $0.44 \mathrm{~mm}$ neutrons from inelastic phonon scattering, the beryllium

\footnotetext{
${ }^{6}$ Bimba model \# T-316-EB2

${ }^{7}$ LND model \# 3005
} 
filter is continuously cooled using liquid nitrogen (LN).

Downstream of the filter is a supermirror polarizer. In a supermirror polarizer, the undesired neutron spin state is reflected out of the direct beam using a a multilayered, $\mathrm{CoFeV}$-coated mirror. At the NIOFa facility neutrons are polarized and analyzed using double ' $\mathrm{V}$ ' cavity supermirrors made by SwissNeutronics. They were designed to achieve a neutron polarization $P_{n} \geq 98 \%$ with minimal physical size for neutrons with wavelengths larger than $0.2 \mathrm{~nm}$. Internally, these double cavity supermirrors are divided into three identical channels. Each of these channels have two back-to-back, double-side coated supermirrors in the shape of a ' $\mathrm{V}$ '. This insures that neutrons passing through the channels always pass through multiple coatings and thus maximize the final neutron polarization. The supermirror has an overall length of $260 \mathrm{~mm}$ with an interior that is $10 \mathrm{~mm}$ wide by $50 \mathrm{~mm}$ tall. A second adjustable slit assembly labeled as Slit2 and identical to Slit1 is used downstream of polarizer to limit the beam divergence. Both Slit1 and Slit2 have a maximum height and width of $76.2 \mathrm{~mm}$. A precession coil spin flipper located after the polarizer can flip the polarity $\left(P_{n} \rightarrow-P_{n}\right)$ of the neutron beam when energized. The interferometer rests on a custom $1.5 \mathrm{~m}$ by $1.8 \mathrm{~m}$ nonmagnetic optical table (see Fig. 10) with $25.4 \mathrm{~mm}$ separated 1/4-20 threaded holes that allows easy sample mounting and detector placement.

Since temperature fluctuations as little as $10 \mathrm{mK}$ can produce phase drifts, an aluminum box $76.2 \mathrm{~cm}$ by $76.2 \mathrm{~cm}$ by $76.2 \mathrm{~cm}$ that is lined with cadmium provides isolation from the environment. This aluminum box is isolated from the breadboard by a $3 \mathrm{~mm}$ thick fiberglass base. PID looped temperature controlled heaters are placed along the walls of the aluminum box. A spin analyzer identical the supermirror polarizer can analyze the outgoing polarization state of the O- or H- beam. A beam block consisting of a combination of high-density polyethylene, boron nitride, and lead is placed at the end of the optical table to completely stop the direct neutron beam. 


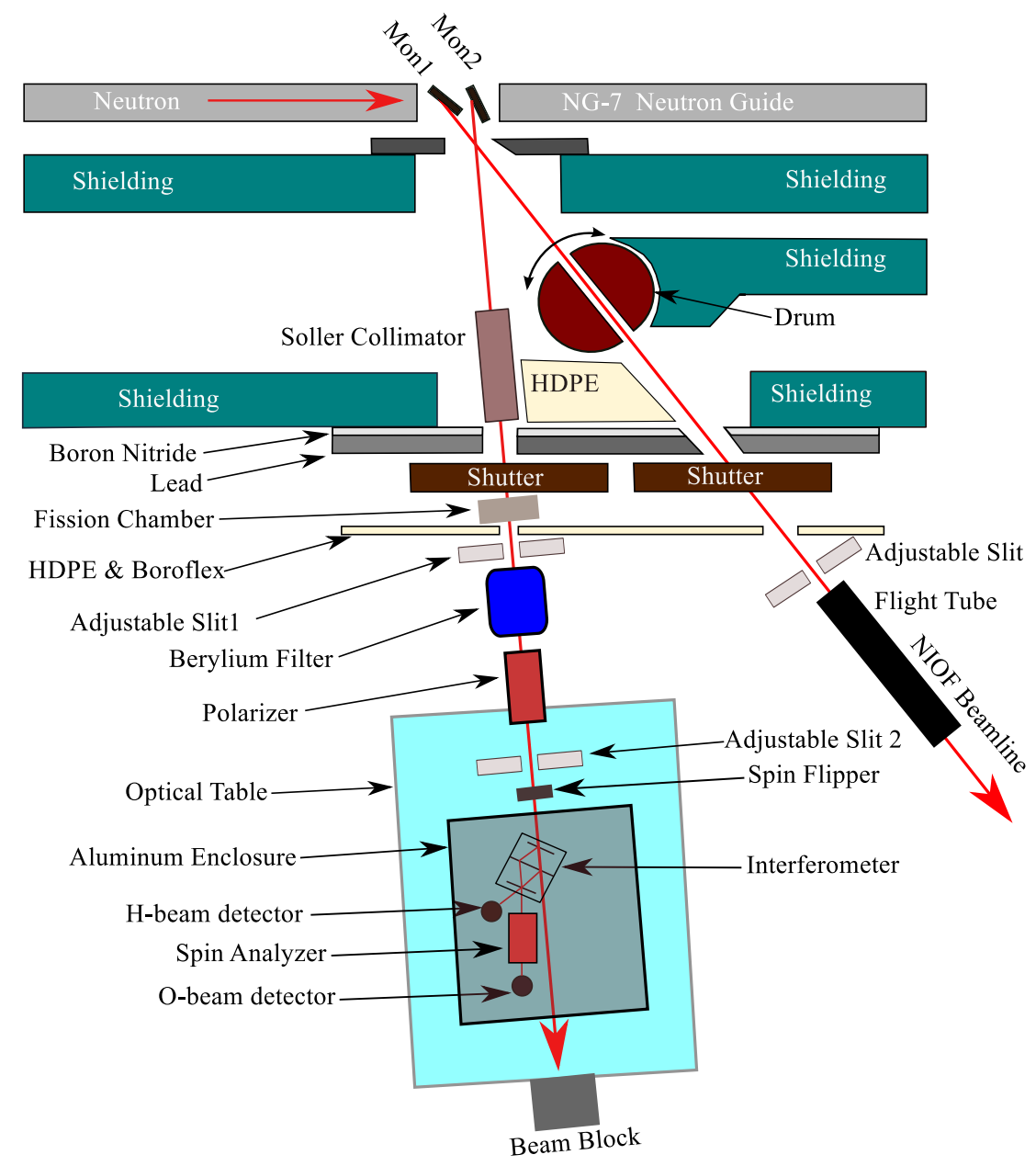

Figure 9: Schematic of the new facility. A PG(002) crystal (Mon2) reflects $0.44 \mathrm{~nm}$ neutrons into the facility. The neutron beam passes through a through collimator, fission chamber, beryllium filter, polarizer and spin flipper before entering a cadmium-lined, aluminum enclosure that houses the the interferometer. The crystal Mon1 reflects neutrons into the NIOF hutch.

The interferometer is supported by a rotation ${ }^{8}$, translation $^{9}$ and height

\footnotetext{
${ }^{8}$ PI Micos model \# PRS-200 180:1 with SiGNUM Encoder

${ }^{9}$ Huber model \# 5101.20
} 


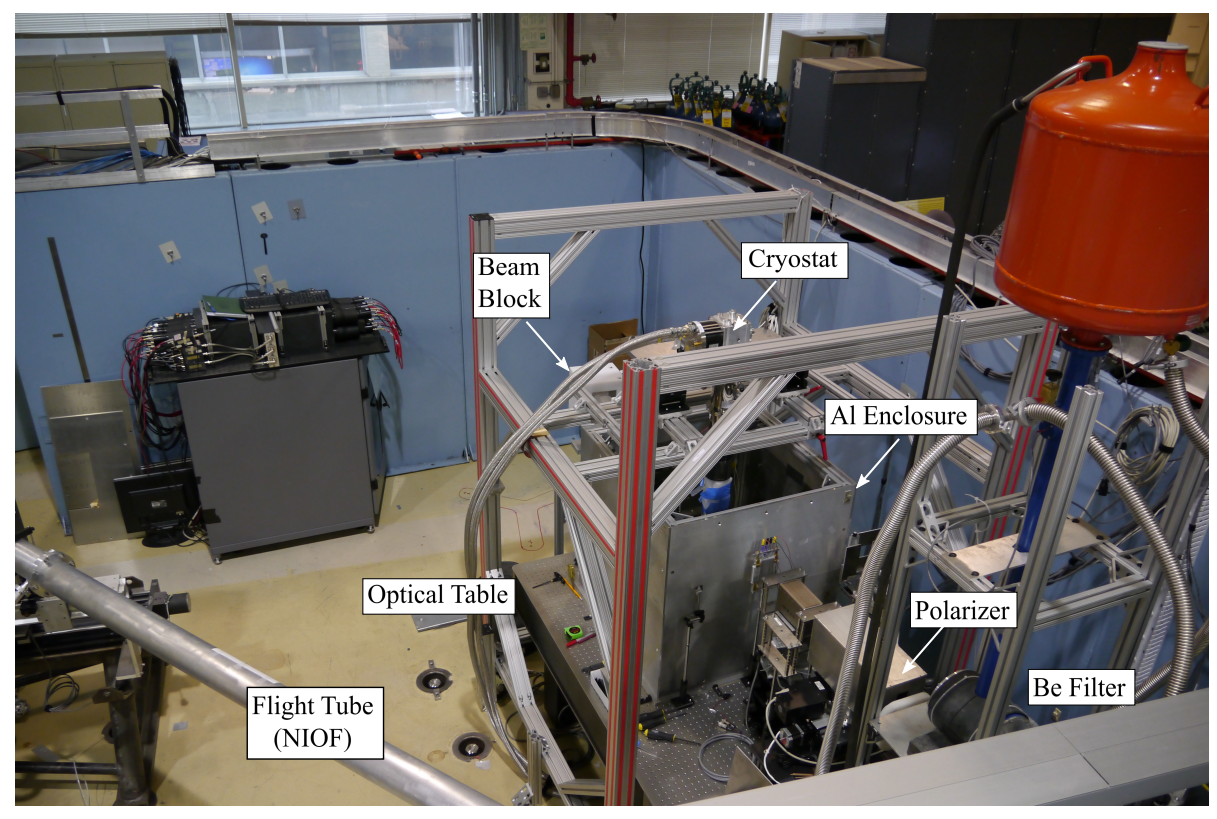

Figure 10: The actual setup of the NIOFa beamline looking from the guide.

stage ${ }^{10}$. The rotation stage is used to find the Bragg angle of the interferometer which is typically $3.5 \times 10^{-3}$ radians wide (full-width-half-max). The translation and the height stage are used to find the interferometer's "sweet" spot; an area typically no bigger than few square millimeters where the contrast is highest. The interferometer crystal rests on a common pool-table felt cloth which, from experience, provides enough friction to hold the interferometer in place without stressing the base of the interferometer. Both the H-beam and O-beam detectors are 10 bar ${ }^{3} \mathrm{He}$ filled proportional counters that are typically $25.4 \mathrm{~mm}$ in diameter ${ }^{11}$ although other size and pressure detectors are sometimes used depending on experimental conditions. 


\subsection{Beam Characteristics}

\subsubsection{Fleuence}

The neutron fluence rate was measured after the first slit (Slit1) at new facility using a calibrated fission chamber containing $32.6 \mu \mathrm{g}$ of Uranium. Data was taken at the different positions relative to the center of the beam. The measured neutron fluence at the center was $3.56 \times 10^{6}$ neutrons $/\left(\mathrm{cm}^{2} \cdot \mathrm{s}\right)$ Table 5 shows the measured fluence rates as a function of beam position relative to the center. This is higher than the measured NIOF neutron fluence rate of $1.32 \times 10^{5}$ neutrons $/\left(\mathrm{cm}^{2} \cdot \mathrm{s}\right)$ at $\lambda=0.235 \mathrm{~nm}$ after its first monochromator. Systematic uncertainties of calibrated fission chamber owing to the deposit particulars are estimated to be $\approx 10 \%$. Fluence rates for both NIOFa and NIOF included neutrons with wavelengths $\lambda / \mathrm{n}$ where $\mathrm{n}=2,3, \ldots$ present in the beam (Fig. 11a). In the case of the NIOFa the fractional component of $\lambda / \mathrm{n}$ compared to the peak $\lambda$ was $25 \%$. To eliminate the faster neutrons, a $76.2 \mathrm{~mm} \times 76.2 \mathrm{~mm}$ $\times 152.4 \mathrm{~mm}$ long berylium filter was installed immediately after the upstream slit assembly. Beryllium scatters neutrons below the Bragg cut-off $\lambda \leq 0.396$ nm out of the direct beam. With the Be filter installed the intensity of the beam dropped $62.2 \%$ overall as measured using a fission chamber but no detectable $\lambda / \mathrm{n}$ could be seen (see Fig. 11b) using a disk chopper and measuring the time-of-flight. Cooling the Be filter to liquid nitrogen temperatures minimizes phonon scattering and thus increases transmission for $0.44 \mathrm{~nm}$ neutrons.

\footnotetext{
${ }^{10}$ Huber model \# 5103.A20

${ }^{11}$ For the $25.4 \mathrm{~mm}$ in diameter: GE Energy, model \# RS-P4-0802-204
}

\begin{tabular}{lr}
$\begin{array}{l}\text { Calibrated detector } \\
\text { position (relative to center) }\end{array}$ & $\begin{array}{r}\text { Fluence rate } \\
\text { (neutrons } /\left(\mathrm{cm}^{2} \cdot \mathrm{s}\right)\end{array}$ \\
\hline \hline $0 \mathrm{~cm}$ & $3.56 \times 10^{6}$ \\
$1 \mathrm{~cm}$ up & $3.12 \times 10^{6}$ \\
$1 \mathrm{~cm}$ down & $2.83 \times 10^{6}$ \\
$0.5 \mathrm{~cm}$ toward NIOF & $2.63 \times 10^{6}$ \\
$0.5 \mathrm{~cm}$ toward reactor & $2.17 \times 10^{6}$ \\
\hline
\end{tabular}

Table 5: Neutron fluence measurement of NIOFa beamline using a calibrated fission chamber at different positions relative to the beam center. 
When the filter was cold the overall drop of intensity was measured to be $15 \%$ when using a fission chamber and corresponds to very little transmission loss — for $0.44 \mathrm{~nm}$ neutrons. The difference between the measured loss of $15 \%$ and the expected $25 \%$ loss is consistent with the efficiencies of the fission chamber at different neutron wavelengths.

\subsubsection{Wavelength Measurement}

The wavelength was analyzed using a nearly perfect (111) silicon crystal analyzer in Laue geometry. This particular crystal is called nearly perfect because it has a relatively small mosaic which allows a greater intensity to be reflected than in a perfect crystal. The analyzer crystal was rotated $\pm \theta_{\mathrm{B}}$ (Fig. 12) using a stage with a highly accurate encoder ${ }^{12}$. The analyzer crystal was also tilted so that the lattice planes could be optimally aligned. Two detectors were placed at angles $\pm 2 \theta_{\mathrm{B}}$ to measure the intensities of parallel and anti-parallel reflected beams and third detector was placed on the direct beam after the analyzer crystal to measure the intensity drop of the transmitted beam. Figure 13 shows the measured $\theta_{\mathrm{B}}$ as a function of tilt angle where the minimum is the correct Bragg angle. By comparing the peak positions of the anti-parallel and parallel reflected beams (see Fig. 14a) in the two detectors and using Eqn. 7 one can determine $\lambda$. The wavelength of new facility was measured to be $\lambda=(0.4389$ $\pm 0.0009) \mathrm{nm}$ with $\Delta \lambda / \lambda \approx 0.8 \%$ (see Fig. 14b).

\subsubsection{Contrast}

The parameter $C$ in Eqn. 8 is known as the contrast or fringe visibility of the interferometer, it is the modulation depth in the intensity of the O-beam with the rotation of the phase flag. Contrast is a measure of interferometer quality and is calculated from the background adjusted ratio

$$
C=\frac{I_{O, \max }-I_{O, \min }}{I_{O, \max }+I_{O, \min }}
$$

\footnotetext{
${ }^{12}$ Heidenhain model \# RON 28718000 lines per revolution
} 
where $I_{O, \max }$ and $I_{O, \min }$ are maximum and minimum intensities of the O-beam, respectively. Contrast of an ideal interferometer is $100 \%$, but in practice the maximum contrast for a neutron interferometer is always less than unity due to reasons such as imperfections in the interferometer, sample absorption, coherence effects, temperature gradients, and environment instabilities. The maximum O-beam contrast of the interferometers at NIST is $\approx 90 \%$. This is not true of the H-beam which, complicated by the exact reflection and transmission coefficients of the interferometer, has $I_{H, \min } \approx I_{O \text {,max }}$. and therefore always has lower contrast than that of the O-beam. Higher contrast suggests higher quality of the neutron interferometer and/or more stable operating environmental conditions. The nonmagnetic optical table at the NIOFa facility has vibration isolation supports to minimize vibrations of the interferometer crystal. Typically neutron interferometers are concerned with vibrations greater than $1 \mathrm{~Hz}$. This low frequency is outside of most standard vibration isolated optical tables. A table with active support structure ${ }^{13}$ was purchased that suppresses vibrations at this level using PZT driven compensators. However well vibrationally damped this system was, under normal operation the absolute position of the table's surface can drift. This is a disadvantage for neutron interferometry since the neutron source (unlike that for light) can't be mounted on the table. For this reason several other support systems where tested. These included a pressuredriven control scheme from the manufacture ${ }^{14}$, a modified feedback system, and supporting the table on simple rubber pads. Figure 15 shows the contrast of an interferometer for different vibration isolation configurations: the optical table is supported in Fig. 15a by air pads and in Fig. 15b by rubber pads. Currently the optical table is supported only by rubber pads. In this configurations an interferometer that in the hutch has $C=90 \%$ at $0.271 \mathrm{~nm}$ decreases to $C=21 \%$ at $0.44 \mathrm{~nm}$. Part of the decrease in contrast is most likely due to the lower neutron velocity as well as that with an increase in Bragg angle the neutron

\footnotetext{
${ }^{13}$ TMC model \# STACIS iX

${ }^{14}$ model \# PEPS II
} 
beam interacts with a larger slice (and thus potentially more imperfections) of the interferometer blades.

\subsubsection{Neutron polarization}

NIOFa beamline is designed for spin based neutron experiments which require a high degree of neutron polarization. Neutrons are polarized and analyzed using double ' $\mathrm{V}$ ' cavity supermirrors ${ }^{15}$. The neutron polarization $P_{n}$ is determined by measuring the flipping ratio $f$ defined as

$$
f=\frac{I_{\text {off }}}{I_{\text {on }}}
$$

where off(on) denotes the state of a precession spin flipper located in between the polarizer and analyzer. The quantity $f$ is related to the polarization by

$$
P_{a} P_{n}=\frac{f-1}{f+1}
$$

where $P_{a}$ is the analyzer efficiency. Since we used the same polarizer and analyzer models it is assumed that $P_{a} \approx P_{n}$. Initially neutron polarization measured at the NIOFa was $\leq 90 \%$ because of the presence of $\sim 26 \% \lambda / \mathrm{n}$ which are left unpolarized. Neutron polarization increased to $\sim 99 \%$ after the installation of beryllium filter. We have found that the exact $P_{n}$ for these polarizers depends very strongly on the experimental conditions such a beam size, divergence and polarizer and analyzer positions.

\section{4. $D A Q$}

For motion control, the new facility uses two 3 -axis controllers ${ }^{16}$ that can operate both 2-phase stepper motors and DC motors, incorporates hard limits, encoders, and drive motors in either closed or open loop operation. For 5-phase motors a 4 -axis controller ${ }^{17}$ is keep in-reserved, but is rarely used. Temperature

\footnotetext{
${ }^{15}$ custom designed and made by SwissNeutronics

${ }^{16}$ Newport model \# 301

${ }^{17}$ Kohzu model \# 410
} 
is PID controlled and monitored ${ }^{18}$ with either platinum resistors or thermistors. All processes and control is administered through a single Windows-based computer. Each communicates to the PC using RS232 communication standards or through virtual COM ports when directly using USB connections .

The detector electronics are all standard NIM modules. Each ${ }^{3} \mathrm{He}$ detector is supplied ${ }^{19}$ a bias voltage of $1200 \mathrm{~V}$. The signal from the ${ }^{3}$ He detectors is transported via a $9.1 \mathrm{~cm}$ long MHV to SHV cable to a preamp ${ }^{20}$ after which the signal travels a few meters to an amplifier $/ \mathrm{SCA}^{21}$ where the signal is converted to a standard TTL pulse. This TTL pulse is registered by a DAQ control board $^{22}$ as a neutron count. Fission chamber neutron detectors use the same electronics but operate at a lower bias voltage of $400 \mathrm{~V}$. Four detectors can be supported simultaneously with this setup. Electrical current for the precession spin flipper is provided by high current supplies ${ }^{23}$ and controlled using a low voltage $^{24}$ DC signal.

Data is saved in either .txt, .csv, or excel-native .xls files. The DAQ is controlled through Visual Basic for Applications (VBA) script and customized excel templates. Excel is an atypical program to run experiments but was chosen because of its universally familiar and expected continuous support from Microsoft. Excel is more than adequate for our needs because data rates at the instrument are modest; requiring no Herculean DAQ software. Remote monitoring and control is accomplished with independent executable programs, written in-house using Visual Basic (VB) or C\# which can be communicated with through VBA script.

\footnotetext{
${ }^{18}$ Lakeshore model \# 331 or 336 and SRS model \# CTC100

${ }^{19}$ Ortec model \# 660

${ }^{20}$ Ortec model \# 142IH

${ }^{21}$ Ortec model \# 590A

${ }^{22} \mathrm{NI}$ model \# PCI-6602

${ }^{23}$ Kepco model \# ATE $30-15$

${ }^{24}$ Labjack model \# U12 DAQ
} 


\section{Future work}

Improvements to the new facility are still ongoing. At this time, only the prototype DFS interferometer exists. Unfortunately with a 220 lattice vector this interferometer can't operate at the peak $0.44 \mathrm{~nm}$ wavelength. Neutron interferometer crystals are exceedingly hard to manufacture as high-quality, perfect silicon ingots are expensive. More importantly expertise in machining silicon for neutron interferometry purposes is being lost with time as the apex in silicon manufacturing research was during the 1970's and 1980's. Despite this, two new DFS-type interferometers designed for $0.44 \mathrm{~nm}$ have been procured by the University of Waterloo. In support of this effort, the University of Waterloo has installed a facility for researching and improving crystal manufacturing techniques. These new vibrationally-insensitive, DFS-type interferometers will greatly improve contrast at the facility.

A test of a cryostat in the neutron beam using a well characterized test sample will soon be completed. Thermal neutron backgrounds at the facility are still high and increased shielding for both the detectors and the facility overall are being implemented. Temperature control has been demonstrated at the beamline but is offline until the DFS-type interferometers are tested. Continuous work on increasing the neutron polarization and spin control are ongoing. We hope that with these improvements the new facility NIOFa can transition from a developing test station to a User-Supported neutron facility.

\section{Conclusion}

The neutron interferometer facility at NIST is a functionally robust yet precise instrument capable of a wide array of both fundamental and applied neutron experiments. The NCNR now supports a second beamline for neutron interferometry applications that has increased flux and certain advantages over the hutch facility. The new NIOFa has improved neutron polarimetry capabilities, increased ease-of-access, a designated cryostat with vibration isolation, and environmental temperature control. Currently low-contrast observed at the 
beamline is being addressed with the construction of new interferometers better suited to work under these conditions. Our hope is that it will greatly expand the interferometry program at NIST in the filed of spin-based neutron interferometry, testing of quantum mechanics, spintronics, neutron spin manipulation, material research and demonstrations of quantum information science.

\section{Acknowledgments}

We are very grateful for the engineering and technical support staff of NCNR and NIST without which this addition would not have been possible. Specifically, we would like to thank D. Pierce for all his work on implementing the guide cut, J. Barker and C. Gagnon for a disk chopper, and G. Balitc for technical assistance. Financial support provided by the NSERC 'Create' and 'Discovery' programs, CERC and the NIST Quantum Information Program, and NSF grant PHY-1205342 is acknowledged. DAQ software is written and maintained by D. L. Jacobson. We greatly appreciate interferometry discussions with S. A. Werner. This work is supported by NIST.

[1] NCNR Accomplishments and Opportunities, the NCNR produces an annual report that can be found online both for the current and previous versions at http://www.ncnr.nist.gov/AnnualReport.

[2] R. E. Williams, J. M. Rowe, Developments in neutron beam devices and an advanced cold source for the NIST research reactor, Physica B311 (1-2) (2002) 117 - 122. doi:DOI: 10.1016/S0921-4526(01)01123-1.

URL http://www.sciencedirect.com/science/article/ B6TVH-44GF300-T/2/98fda38eeca8296e5a330ca28e8f28ab

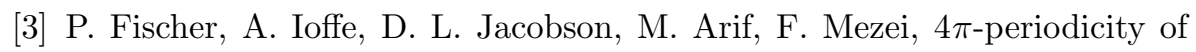
the spinor wave function under space rotation, Nucl. Inst. Meth. A440 (3) (2000) 575 - 578. doi:DOI: 10.1016/S0168-9002(99)01039-6.

URL http://ww.sciencedirect.com/science/article/ B6T JM-3YJ9CDG-N/2/3c9cc9606e88858f c1bb6ba4637d659f 
[4] P. R. Huffman, D. L. Jacobson, K. Schoen, M. Arif, T. C. Black, W. M. Snow, S. A. Werner, Precision neutron interferometric measurement of the $n-{ }^{3} \mathrm{He}$ coherent neutron scattering length, Phys. Rev. C70 (1) (2004) 014004 .

URL http://link.aps.org/abstract/PRC/v70/e014004

[5] A. Ioffe, M. Arif, D. L. Jacobson, F. Mezei, Precision neutron inferferometric search for evidence of nuclear quantum entanglement in liquid $\mathrm{H}_{2} \mathrm{O}-\mathrm{D}_{2} \mathrm{O}$ mixtures, Phys. Rev. Lett. 82 (11) (1999) 2322-2325. doi:10.1103/PhysRevLett.82.2322.

[6] K. Schoen, D. L. Jacobson, M. Arif, P. R. Huffman, T. C. Black, W. M. Snow, S. K. Lamoreaux, H. Kaiser, S. A. Werner, Precision neutron interferometric measurements and updated evaluations of the $n-p$ and $n-d$ coherent neutron scattering lengths, Phys. Rev. C67 (4) (2003) 044005. doi:10.1103/PhysRevC.67.044005.

[7] M. G. Huber, M. Arif, W. C. Chen, T. R. Gentile, D. S. Hussey, T. C. Black, D. A. Pushin, C. B. Shahi, F. E. Wietfeldt, L. Yang, Neutron interferometric measurement of the scattering length difference between the triplet and singlet states of $n-{ }^{3}$ He, Phys. Rev. C 90 (2014) 064004. doi:10.1103/PhysRevC.90.064004.

URL http://link.aps.org/doi/10.1103/PhysRevC.90.064004

[8] D. A. Pushin, M. Arif, M. G. Huber, D. G. Cory, Measurements of the vertical coherence length in neutron interferometry, Phys. Rev. Lett. 100 (25) (2008) 250404. doi:10.1103/PhysRevLett.100.250404.

URL http://link.aps.org/abstract/PRL/v100/e250404

[9] C. J. Wood, M. O. Abutaleb, M. G. Huber, M. Arif, D. G. Cory, D. A. Pushin, Quantum correlations in a noisy neutron interferometer, Phys. Rev. A 90 (2014) 032315. doi:10.1103/PhysRevA.90.032315. URL http://link.aps.org/doi/10.1103/PhysRevA.90.032315 
[10] D. L. Jacobson, M. Arif, L. Bergmann, A. Ioffe, Development of the neutron phase contrast imaging technique and its application in material science research, EUV, X-Ray, and Neutron Optics and Sources 3767 (1) (1999) 328-335. doi:10.1117/12.371131.

URL http://link.aip.org/link/?PSI/3767/328/1

[11] D. A. Pushin, D. G. Cory, M. Arif, D. L. Jacobson, M. G. Huber, Reciprocal space approaches to neutron imaging, Appl. Phys. Lett. 90 (22) (2007) 224104. doi:10.1063/1.2737390.

URL http://link.aip.org/link/?APL/90/224104/1

[12] W. E. Wallace, D. L. Jacobson, M. Arif, A. Ioffe, Application of neutron interferometry to the measurement of thin film density, Applied Physics Letters 74 (3) (1999) 469-471. doi:http://dx.doi.org/10.1063/1.123038. URL http://scitation.aip.org/content/aip/journal/apl/74/3/10. $1063 / 1.123038$

[13] M. Arif, M. S. Dewey, G. L. Greene, D. Jacobson, S. Werner, X-ray determination of the elastic deformation of a perfect crystal neutron interferometer: implications for gravitational phase shift experiments, Phys. Lett. A184 (2) (1994) 154 - 158. doi:DOI: 10.1016/0375-9601(94)90768-4. URL http://www.sciencedirect.com/science/article/ B6TVM-46SNJY4-HN/2/bd5b49048d2bd3a74116a12ddb870f 20

[14] A. Ioffe, D. L. Jacobson, M. Arif, M. Vrana, S. A. Werner, P. Fischer, G. L. Greene, F. Mezei, Precision neutron-interferometric measurement of the coherent neutron-scattering length in silicon, Phys. Rev. A58 (2) (1998) 1475-1479. doi:10.1103/PhysRevA.58.1475.

[15] T. C. Black, P. R. Huffman, D. L. Jacobson, W. M. Snow, K. Schoen, M. Arif, H. Kaiser, S. K. Lamoreaux, S. A. Werner, Precision neutron interferometric measurement of the $n d$ coherent neutron scattering length and consequences for models of three-nucleon forces, Phys. Rev. Lett. 90 (19) (2003) 192502. doi:10.1103/PhysRevLett.90.192502. 
[16] D. A. Pushin, M. Arif, D. G. Cory, Decoherence-free neutron interferometry, Phys Rev 79 (5) (2009) 053635. doi:10.1103/PhysRevA.79.053635. URL http://link.aps.org/abstract/PRA/v79/e053635

[17] D. Pushin, M. Huber, M. Arif, D. Cory, Experimental Realization of Decoherence-Free Subspace in Neutron Interferometry, Phys.Rev.Lett. 107 (2011) 150401. doi:10.1103/PhysRevLett.107.150401.

[18] M. G. Huber, M. Arif, T. C. Black, W. C. Chen, T. R. Gentile, D. S. Hussey, D. A. Pushin, F. E. Wietfeldt, L. Yang, Precision measurement of the n-[sup 3] he incoherent scattering length using neutron interferometry, Physical Review Letters 102 (20) (2009) 200401. doi:10.1103/PhysRevLett.102.200401. URL http://link.aps.org/abstract/PRL/v102/e200401

[19] M. O. Abutaleb, D. A. Pushin, M. G. Huber, C. F. Majkrzak, M. Arif, , D. G. Cory, Design of remnant magnetization fecov films as compact, heatless neutron spin rotators, Applied Physics Letters 101 (2012) 182404. doi:http://dx.doi.org/10.1063/1.4765069.

[20] C. W. Clark, R. Barankov, M. G. Huber, M. Arif, D. G. Cory, D. A. Pushin, Controlling neutron orbital angular momentum, Nature 525 (2015) 504-506.

[21] M. Arif, D. E. Brown, G. L. Greene, R. Clothier, K. Littrell, Multistage position-stabilized vibration isolation system for neutron interferometry, Vibr. Monit. Cont. 2264 (1) (1994) 20-26.

URL http://link.aip.org/link/?PSI/2264/20/1

[22] M. Huber, et al., in preparation.

[23] H. Rauch, S. Werner, Neutron Interferometry: Lessons in Experimental Quantum Mechanics, Clarendon Press, 2000.

[24] K. Olive, P. D. Group, Review of particle physics, Chinese Physics C 38 (9) (2014) 090001. doi:doi:10.1088/1674-1137/38/9/090001.

URL http://stacks.iop.org/1674-1137/38/i=9/a=090001 
[25] E. Feenberg, The scattering of slow electrons by neutral atoms, Phys. Rev. 40 (1) (1932) 40-54.

URL 10.1103/PhysRev.40.40

[26] P. J. Mohr, B. N. Taylor, D. B. Newell, Codata recommended values of the fundamental physical constants: 2006, Rev. Mod. Phys. 80 (2) (2008) 633-730. doi:10.1103/RevModPhys.80.633.

[27] G. Badurek, H. Rauch, J. Summhammer, Time-dependent superposition of spinors, Phys. Rev. Lett. 51 (12) (1983) 1015-1018. doi:10.1103/PhysRevLett.51.1015.

[28] H. Bartosik, J. Klepp, C. Schmitzer, S. Sponar, A. Cabello, H. Rauch, Y. Hasegawa, Experimental test of quantum contextuality in neutron interferometry, Phys. Rev. Lett. 103 (2009) 040403. doi:10.1103/PhysRevLett.103.040403.

URL http://link.aps.org/doi/10.1103/PhysRevLett.103.040403

[29] J. Summhammer, G. Badurek, H. Rauch, U. Kischko, A. Zeilinger, Direct observation of fermion spin superposition by neutron interferometry, Phys. Rev. A27 (5) (1983) 2523-2532. doi:10.1103/PhysRevA.27.2523.

[30] B. E. Allman, H. Kaiser, S. A. Werner, A. G. Wagh, V. C. Rakhecha, J. Summhammer, Observation of geometric and dynamical phases by neutron interferometry, Phys. Rev. A 56 (1997) 4420-4439. doi:10.1103/PhysRevA.56.4420.

URL http://link.aps.org/doi/10.1103/PhysRevA.56.4420

[31] H. Rauch, A. Zeilinger, G. Badurek, A. Wilfing, W. Bauspiess, U. Bonse, Verification of coherent spinor rotation of fermions, Phys. Lett. A54 (6) (1975) 425 - 427. doi:DOI: 10.1016/0375-9601(75)90798-7.

URL http://www.sciencedirect.com/science/article/ B6TVM-46TYS59-K3/2/2acd89455ef 15d54565bb54c9cdd5aae 
[32] R. Colella, A. W. Overhauser, S. A. Werner, Observation of gravitationally induced quantum interference, Phys. Rev. Lett. 34 (23) (1975) 1472-1474. doi:10.1103/PhysRevLett.34.1472.

[33] S. A. Werner, J. L. Staudenmann, R. Colella, Effect of earth's rotation on the quantum mechanical phase of the neutron, Phys. Rev. Lett. 42 (17) (1979) 1103-1106. doi:10.1103/PhysRevLett.42.1103.

[34] A. Cimmino, G. I. Opat, A. G. Klein, H. Kaiser, S. A. Werner, M. Arif, R. Clothier, Observation of the topological Aharonov-Casher phase shift by neutron interferometry, Phys. Rev. Lett. 63 (4) (1989) 380-383. doi:10.1103/PhysRevLett.63.380.

[35] B. E. Allman, A. Cimmino, A. G. Klein, G. I. Opat, H. Kaiser, S. A. Werner, Scalar Aharonov-Bohm experiment with neutrons, Phys. Rev. Lett. 68 (16) (1992) 2409-2412. doi:10.1103/PhysRevLett.68.2409.

[36] A. G. Wagh, V. C. Rakhecha, J. Summhammer, G. Badurek, H. Weinfurther, B. Allman, H. Kaiser, K. Hamacher, D. Jacobson, S. Werner, Neutron interferometric separation of geometric and dynamical phases, J. Phys. Soc. Jap 65.

[37] Certain trade names and company products are mentioned in the text or identified in an illustration in order to adequately specify the experimental procedure and equipment used. In no case does such identification imply recommendation or endorsement by the National Institute of Standards and Technology, nor does it imply that the products are necessarily the best available for the purpose.

[38] C. J. Carlile, P. D. Hey, B. Mack, High-efficiency soller slit collimators for thermal neutrons, Journal of Physics E: Scientific Instruments 10 (5) (1977) 543.

URL http://stacks.iop.org/0022-3735/10/i=5/a=035 


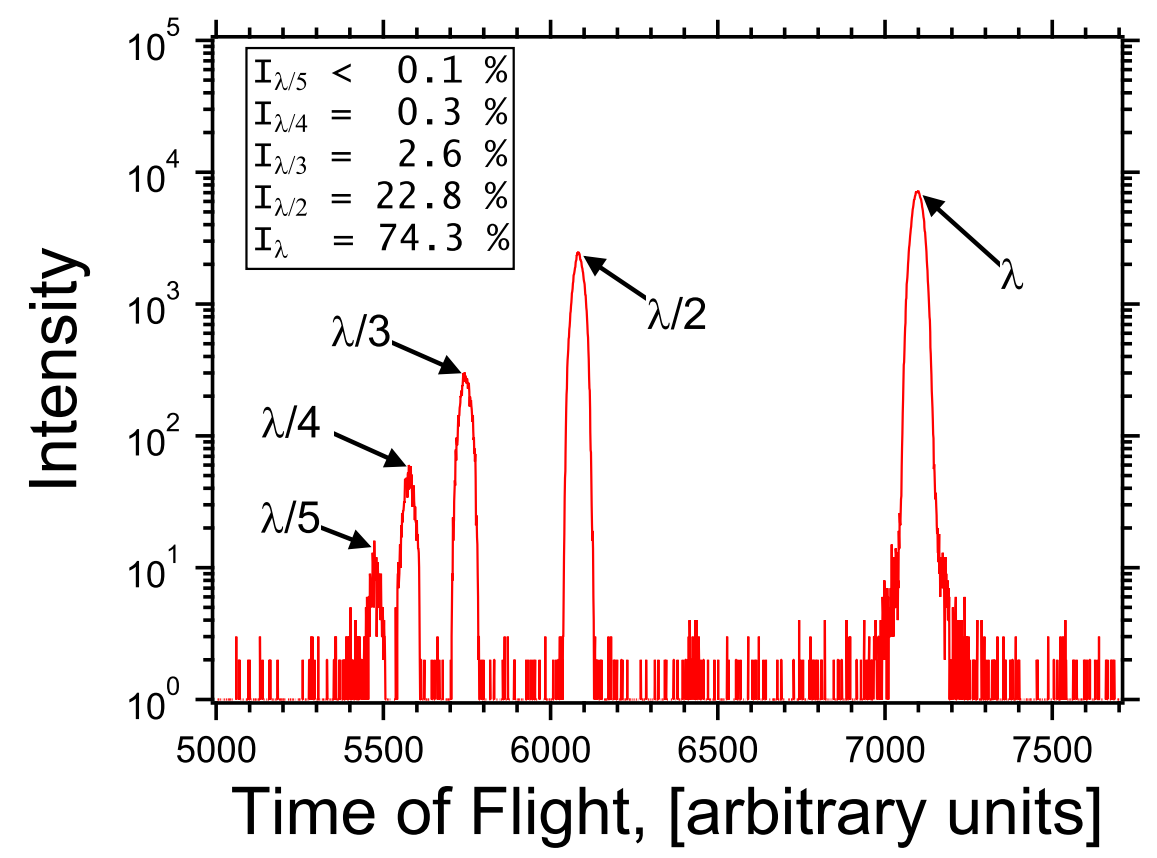

(a)

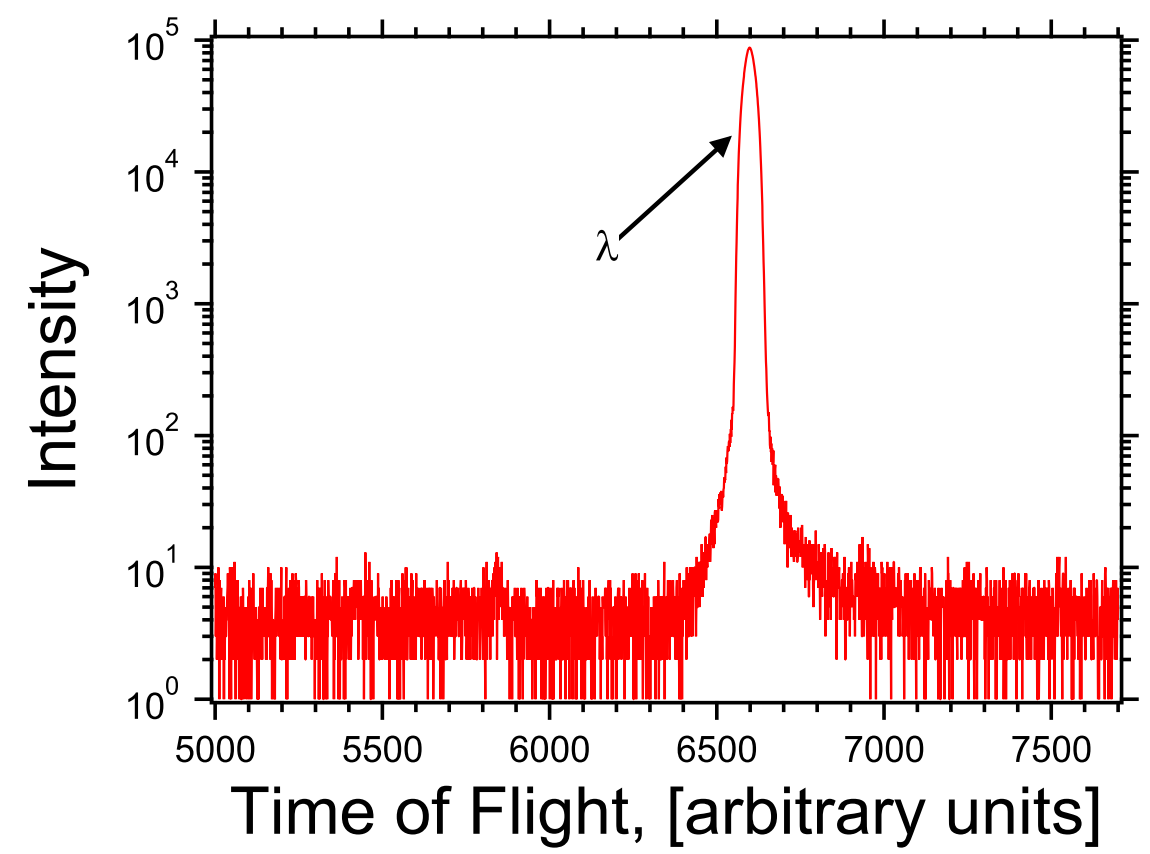

(b)

Figure 11: (a) The neutron spectrum $\lambda=(0.44 / \mathrm{n})$ nm where $\mathrm{n}=1,2,3, \ldots$ measured using a disc chopper before installation of the beryllium filter. Detector efficiencies $\epsilon_{\lambda}$ are a function of neutron wavelength have not been accounted for here. (b) After the installation beryllium filter only the $\lambda=0.44 \mathrm{~nm}$ peak remains. NoBA, measurement time was not the same for (a) and (b). 


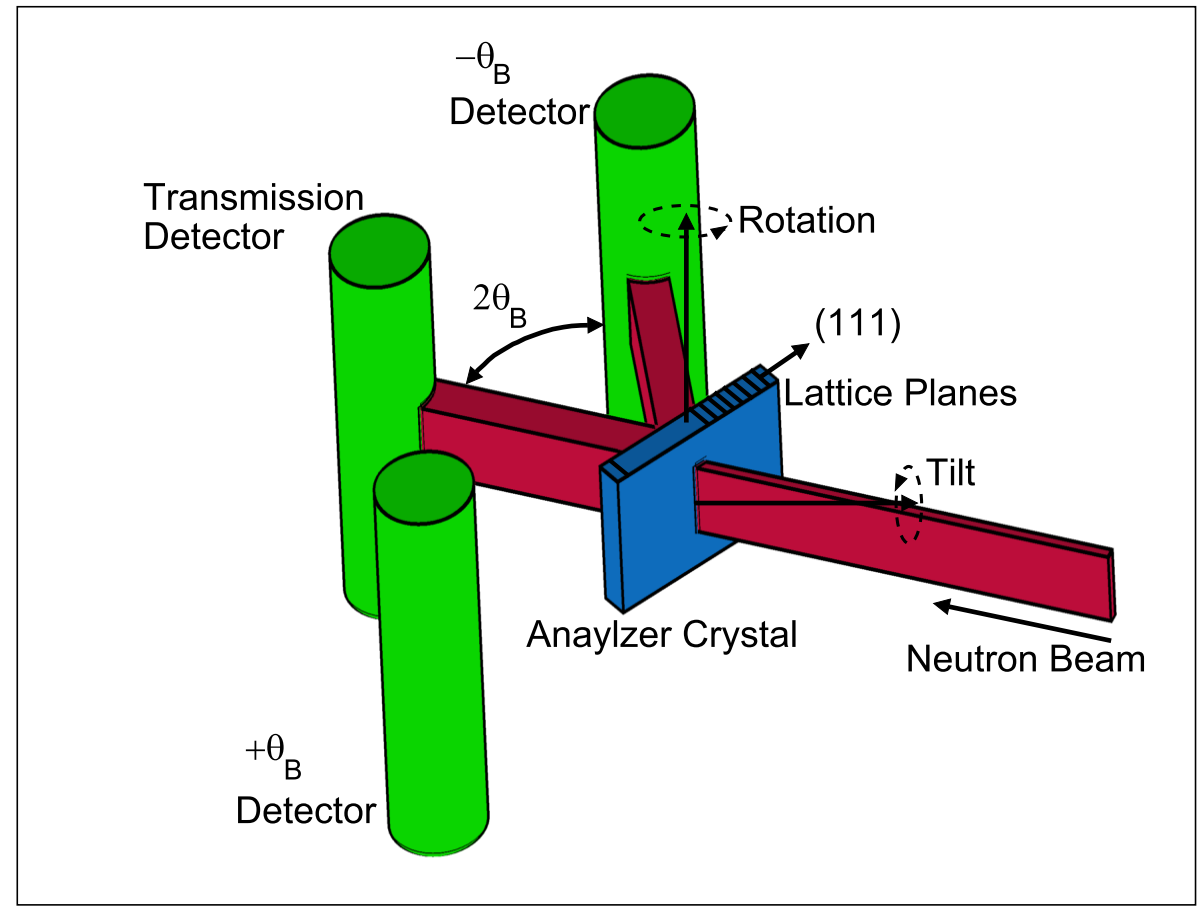

Figure 12: Wavelength measurement setup for the NIOFa beamline. A nearly perfect (111) silicon crystal analyzer was rotated $\pm \theta_{\mathrm{B}}$. Two detectors were placed at $\pm 2 \theta_{\mathrm{B}}$ to measure the reflected intensities. A third detector was placed on the direct beam right after the crystal to measure the decrease in the intensity of transmitted beam. 


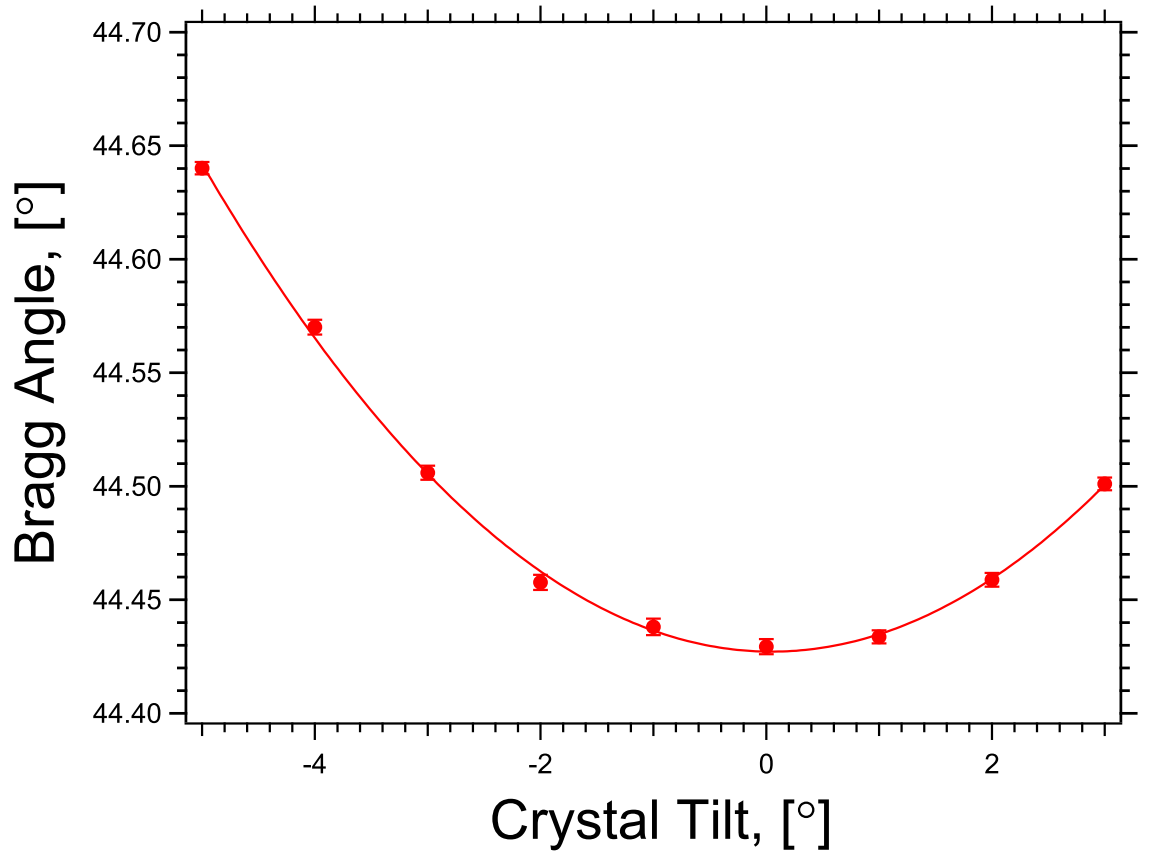

Figure 13: Alignment of a nearly perfect (111) silicon crystal analyzer. 


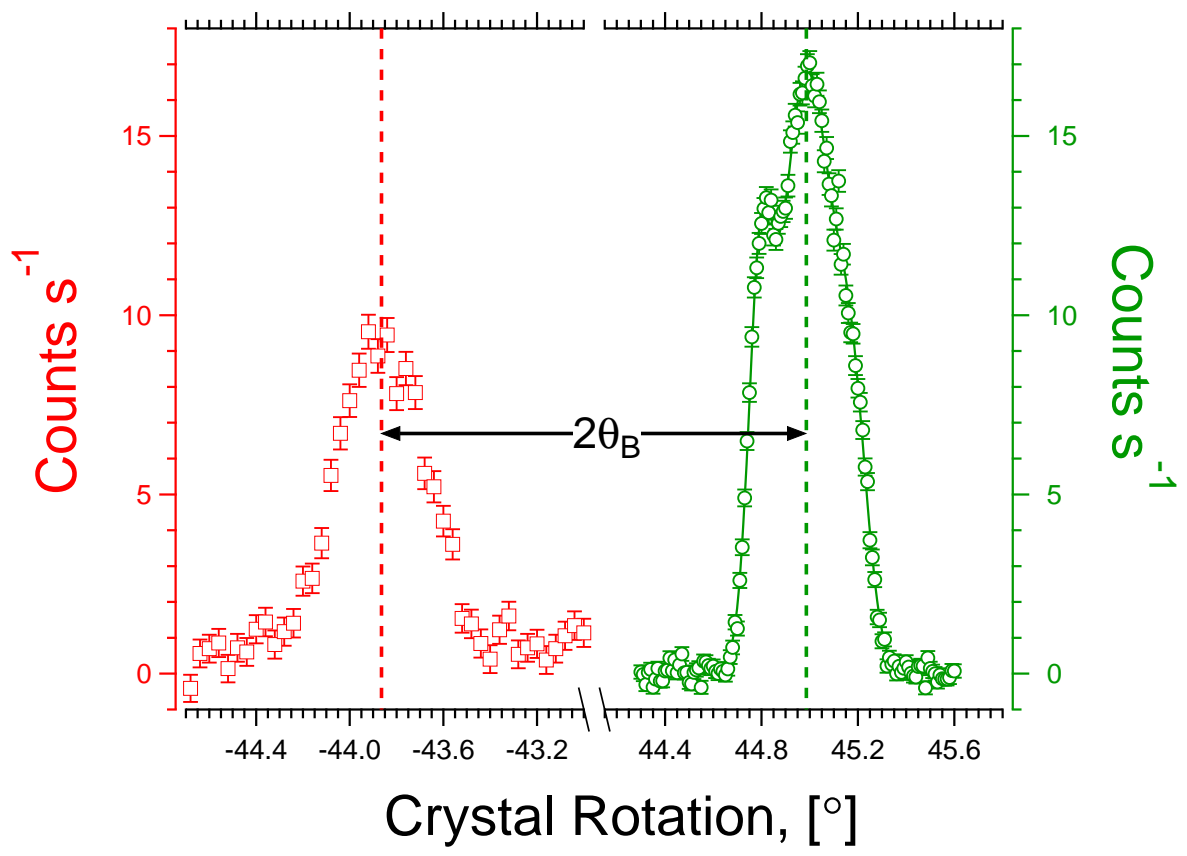

(a)

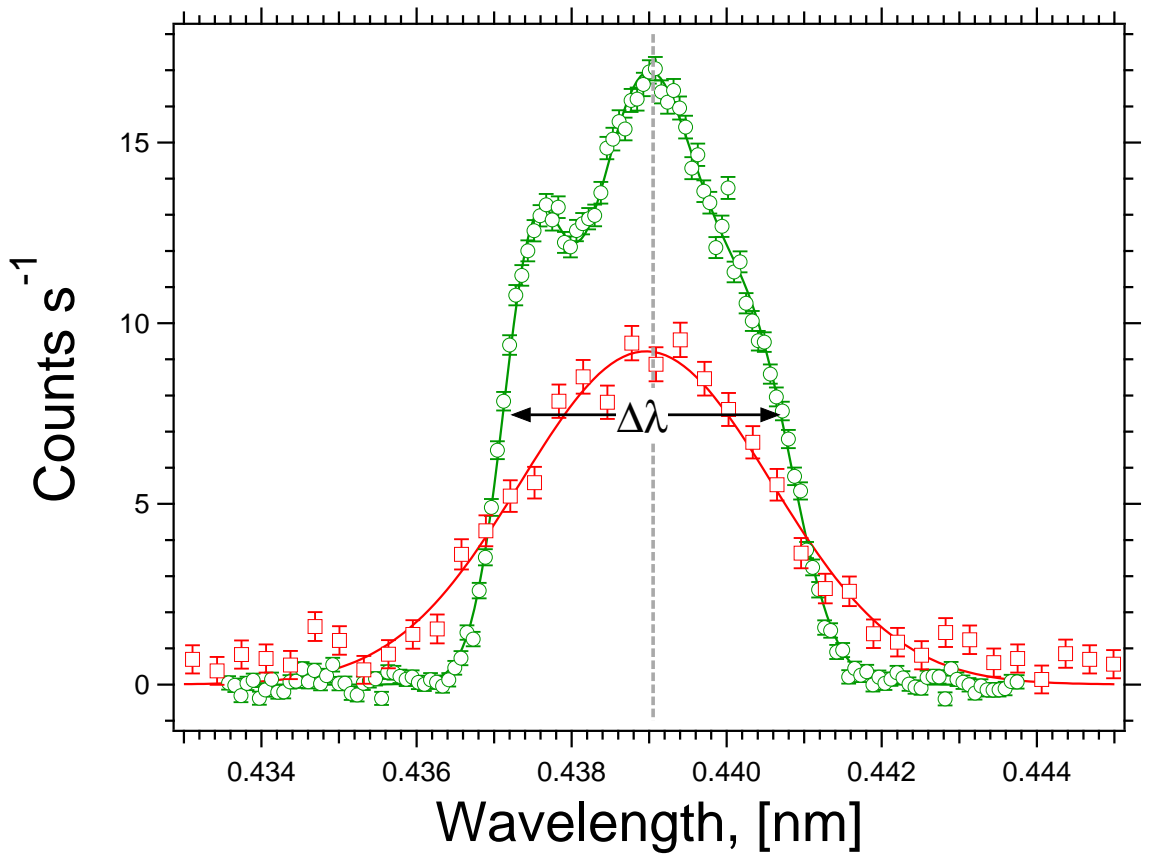

(b)

Figure 14: (a) The anti-parallel (red squares-scale on left) and parallel (green circles-scale on right) Bragg reflections. The average background rate has been subtracted from the points shown. For the parallel reflection the central location was found by taking a weighted average of peak locations of separate three Gaussian 3innctions used to fit the data. (b) Data in (a) plotted versus wavelength. 


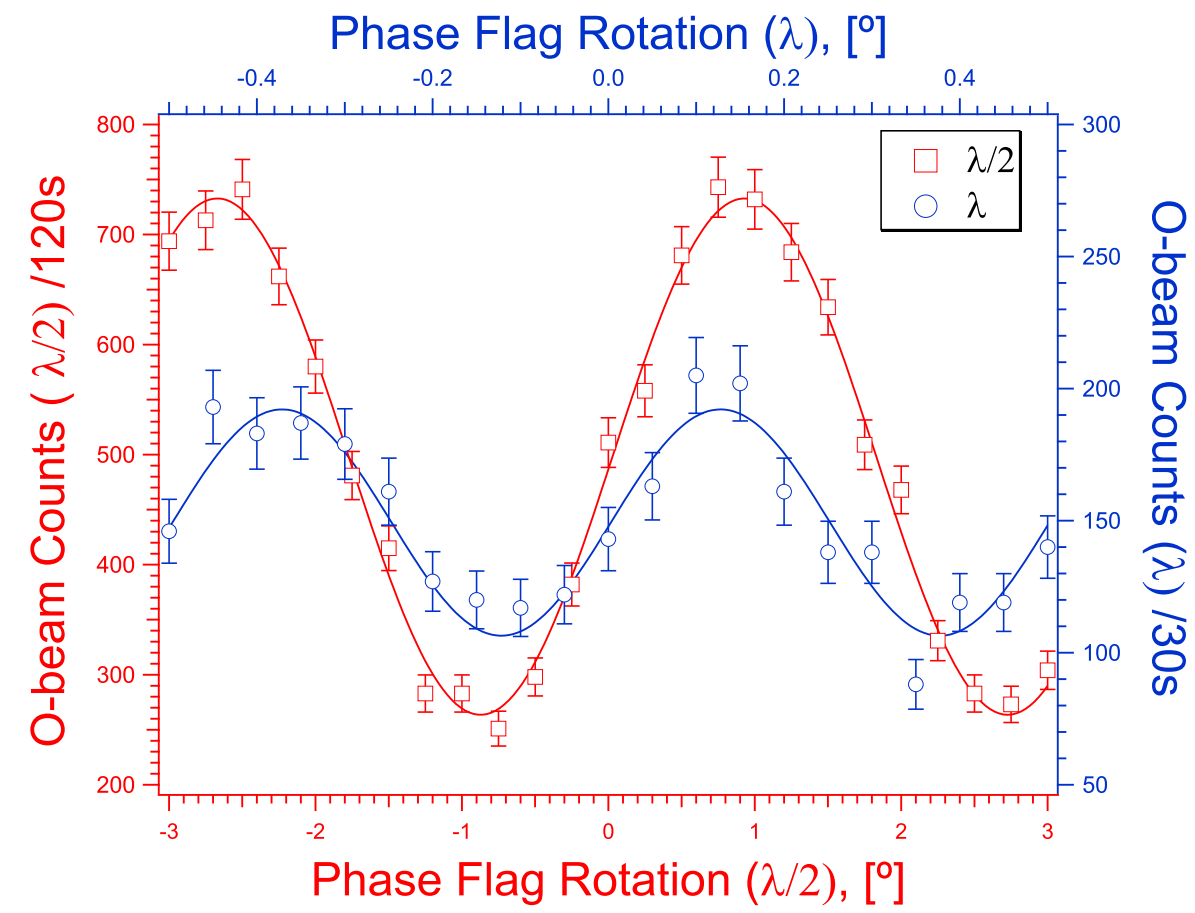

(a)

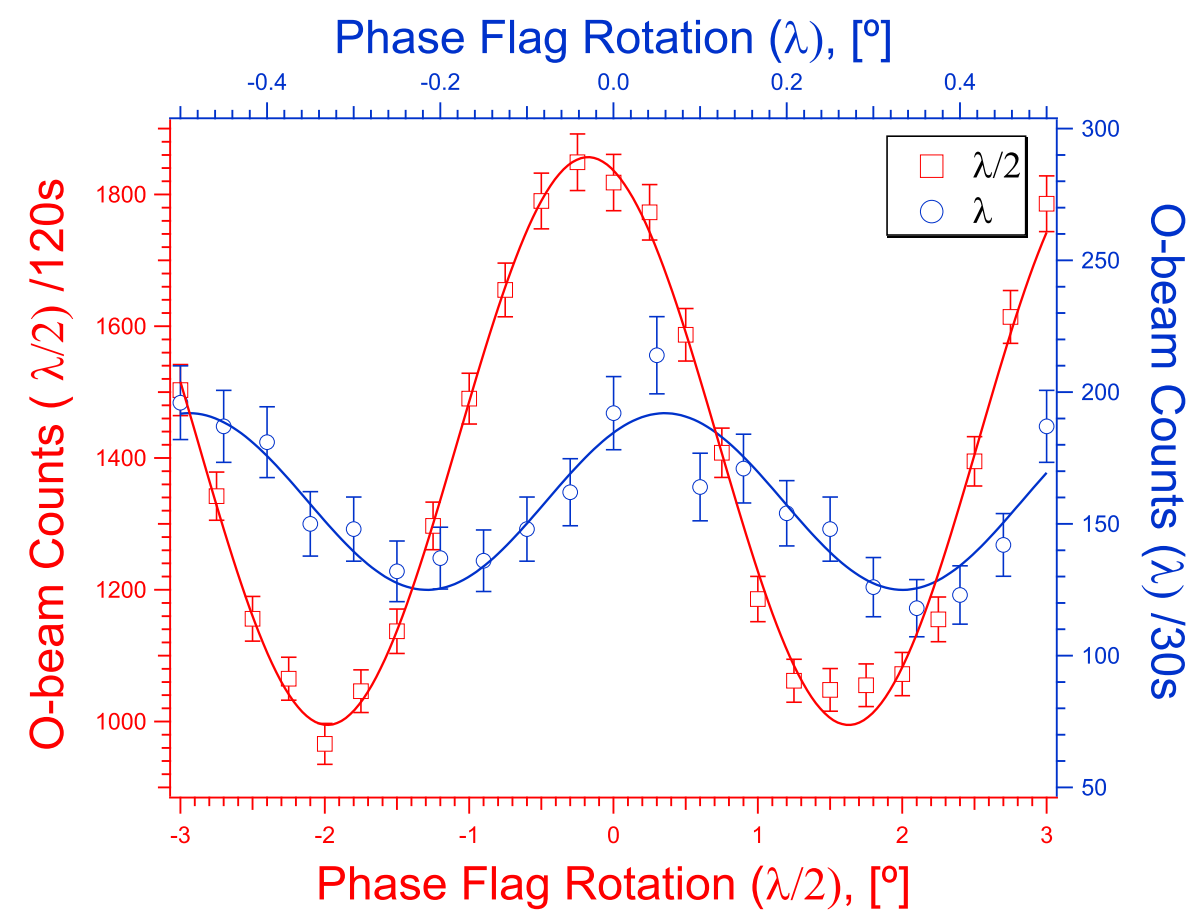

(b)

Figure 15: O-beam intensities for $\lambda=0.22 \mathrm{~nm}$ (red squares) and $\lambda=0.44 \mathrm{~nm}$ (blue circles) neutrons. (a) Here the PZT-control of the STACIS system (which caused positional drift) was turned off, but the table still had some vibration isolation. The contrast was $C=47 \%$ for $0.22 \mathrm{~nm}$ and $C=32 \%$ for $0.44 \mathrm{~nm}$. (b) Without any active vibration isolation. The optical table resting on rubber pads where the contrast decreased to $30 \%$ for $0.22 \mathrm{~nm}$ and $21 \%$ for $0.44 \mathrm{~nm}$. 